\title{
Nobel Lecture: Semiconducting and metallic polymers: The fourth generation of polymeric materials*
}

\author{
Alan J. Heeger \\ Department of Physics, Materials Department, Institute for Polymers and Organic Solids, \\ University of California, Santa Barbara, Santa Barbara, California 93106
}

(Published 20 September 2001)

\section{INTRODUCTION}

In 1976, Alan MacDiarmid, Hideki Shirakawa, and I, together with a talented group of graduate students and postdoctoral researchers, discovered conducting polymers and the ability to dope these polymers over the full range from insulator to metal (Chiang et al., 1977; Shirakawa et al., 1977). This was particularly exciting because it created a new field of research on the boundary between chemistry and condensed-matter physics, and because it created a number of opportunities:

- Conducting polymers opened the way to progress in understanding the fundamental chemistry and physics of $\pi$-bonded macromolecules;

- Conducting polymers provided an opportunity to address questions that had been of fundamental interest to quantum chemistry for decades:

Is there bond alternation in long-chain polyenes?

What is the relative importance of the electronelecron and the electron-lattice interactions in $\pi$-bonded macromolecules?

- Conducting polymers provided an opportunity to address fundamental issues of importance to condensedmatter physics as well, including, for example, the metalinsulator transition as envisioned by Neville Mott and Philip Anderson and the instability of one-dimensional metals discovered by Rudolph Peierls (the "Peierls Instability").

- Finally-and perhaps most important-conducting polymers offered the promise of achieving a new generation of polymers: Materials which exhibit the electrical and optical properties of metals or semiconductors and which retain the attractive mechanical properties and processing advantages of polymers.

Thus, when asked to explain the importance of the discovery of conducting polymers, I offer two basic answers:

(i) They did not (could not?) exist.

(ii) They offer a unique combination of properties not available from any other known materials.

The first expresses an intellectual challenge; the second expresses a promise for utiltity in a wide variety of applications.

\footnotetext{
*The 2000 Nobel Prize in Chemistry was shared by Alan J. Heeger, Alan G. MacDiarmid, and Hideki Shirakawa. This lecture is the text of Professor Heeger's address on the occasion of the award.
}

Conducting polymers are the most recent generation of polymers (Rånby, 1993). Polymeric materials in the form of wood, bone, skin, and fibers have been used by man since prehistoric time. Although organic chemistry as a science dates back to the eighteenth century, polymer science on a molecular basis is a development of the twentieth century. Hermann Staudinger developed the concept of macromolecules during the 1920s. Staudinger's proposal was openly opposed by leading scientists, but the data eventually confirmed the existence of macromolecules. Staudinger was awarded the Nobel Prize in Chemistry in 1953 "for his discoveries in the field of macromolecular chemistry." While carrying out basic research on polymerization reactions at the DuPont Company, Wallace Carothers invented nylon in 1935. Carothers's research showed the great industrial potential of synthetic polymers, a potential that became reality in a remarkably short time. Today, synthetic polymers are used in larger quantities than any other class of materials (larger by volume and larger by weight, even though such polymers have densities close to unity). Polymer synthesis in the 1950s was dominated by Karl Ziegler and Giulio Natta, whose discoveries of polymerization catalysts were of great importance for the development of the modern "plastics" industry. Ziegler and Natta were awarded the Nobel Prize in Chemistry in 1963 "for their discoveries in the field of the chemistry and technology of high polymers." Paul Flory was the next "giant" of polymer chemistry; he created modern polymer science through his experimental and theoretical studies of macromolecules. His insights and deep understanding of macromolecular phenomena are summarized in his book, Principles of Polymer Chemistry, published in 1953 and still useful (and used) today. Flory was awarded the Nobel Prize in Chemistry in 1974 "for his fundamental achievements, both theoretical and experimental, in the physical chemistry of macromolecules."

Because the saturated polymers studied by Staudinger, Flory, Ziegler, and Natta are insulators, they were viewed as uninteresting from the point of view of electronic materials. Although this is true for saturated polymers (in which all of the four valence electrons of carbon are used up in covalent bonds), in conjugated polymers the electronic configuration is fundamentally different. In conjugated polymers, the chemical bonding leads to one unpaired electron (the $\pi$ electron) per carbon atom. Moreover, $\pi$ bonding, in which the carbon orbitals are in the $s p^{2} p_{z}$ configuration and in which the 


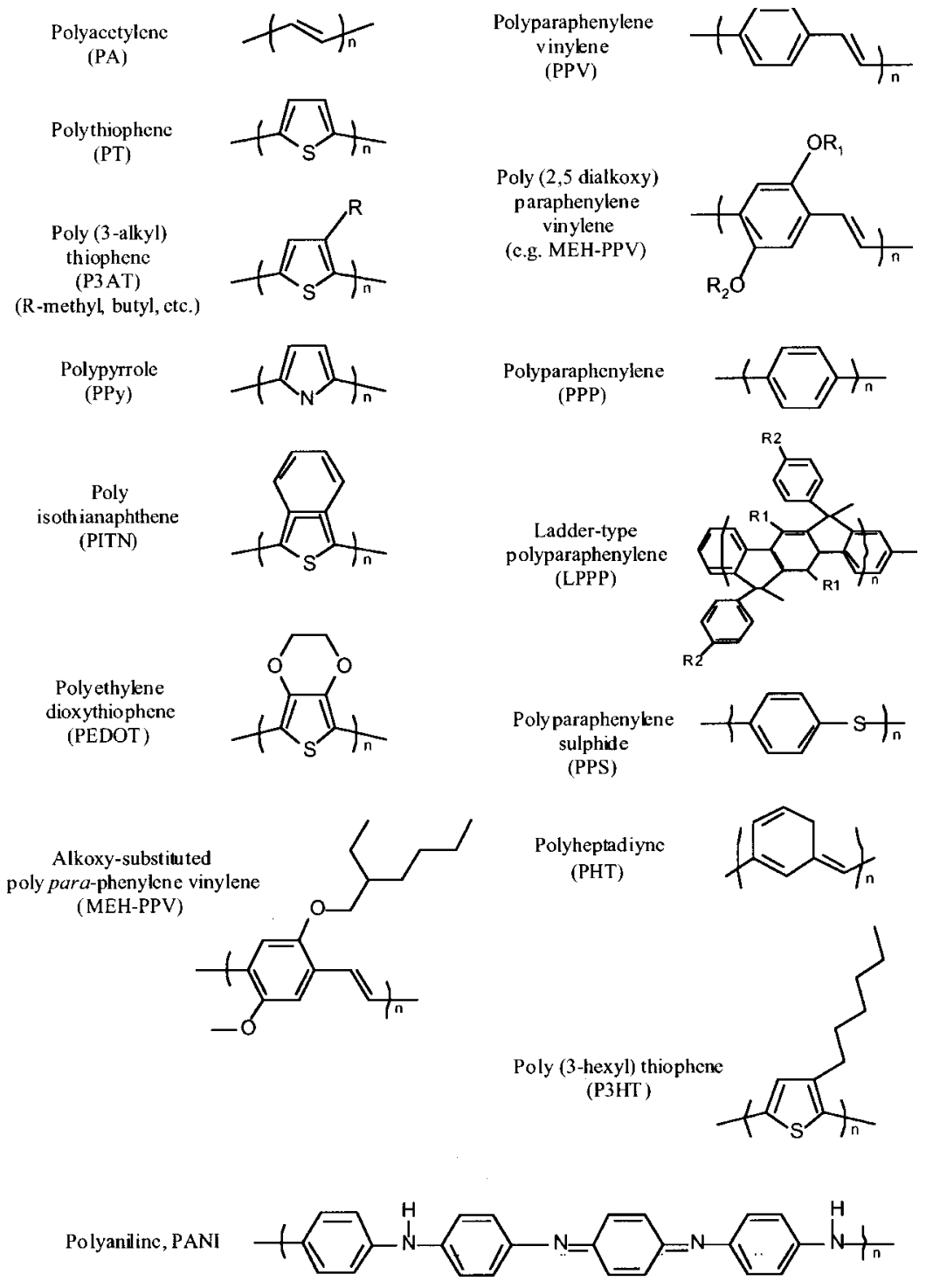

FIG. 1. Molecular structures of examples of conjugated polymers. Note the bondalternated structures. orbitals of successive carbon atoms along the backbone overlap, leads to electron delocalization along the backbone of the polymer. This electronic delocalization provides the "highway" for charge mobility along the backbone of the polymer chain.

As a result, therefore, the electronic structure in conducting polymers is determined by the chain symmetry (i.e., the number and kind of atoms within the repeat unit), with the result that such polymers can exhibit semiconducting or even metallic properties. In his lecture at the Nobel Symposium (NS-81) in 1991, Professor Bengt Rånby designated electrically conducting polymers as the "fourth generation of polymeric materials" (Rånby, 1993).

The classic example is polyacetylene, $(-\mathrm{CH})_{n}$, in which each carbon is $\sigma$ bonded to only two neighboring carbons and one hydrogen atom with one $\pi$ electron on each carbon. If the carbon-carbon bond lengths were equal, the chemical formula, $(-\mathrm{CH})_{n}$ with one unpaired electron per formula unit, would imply a metallic state. Alternatively, if the electron-electron interactions were too strong, $(-\mathrm{CH})_{n}$ would be an antiferromagnetic Mott insulator. The easy conversion to the metallic state on doping (Chiang et al., 1977; Shirakawa et al., 1977) together with a variety of studies of the neutral polymer have eliminated the antiferromagnetic Mott insulator as a possibility.

In real polyacetylene, the structure is dimerized as a result of the Peierls instability with two carbon atoms in the repeat unit, $(-\mathrm{CH}=\mathrm{CH})_{n}$. Thus the $\pi$ band is divided into $\pi$ and $\pi^{*}$ bands. Since each band can hold two electrons per atom (spin up and spin down), the $\pi$ band is filled and the $\pi^{*}$ band is empty. The energy difference between the highest occupied state in the $\pi$ band and the lowest unoccupied state in the $\pi^{*}$ band is the $\pi-\pi^{*}$ energy gap $E_{g}$. The bond-alternated structure of polyacetylene is characteristic of conjugated polymers (see Fig. 1). Consequently, since there are no partially filled bands, conjugated polymers are typically semiconductors. Because $E_{g}$ depends upon the molecular structure of the repeat unit, synthetic chemists are provided with the opportunity and the challenge to control the energy gap by design at the molecular level.

Although initially built upon the foundations of quantum chemistry and condensed-matter physics, it soon became clear that entirely new concepts were involved in 
the science of conducting polymers. The discovery of nonlinear excitations in this class of polymers, solitons in systems in which the ground state is degenerate and confined soliton pairs (polarons and bipolarons) in systems in which the ground-state degeneracy has been lifted by the molecular structure, opened entirely new directions for the study of the interconnection of chemical and electronic structure. The spin-charge separation characteristic of solitons and the reversal of the spin-charge relationship (relative to that expected for electrons as fermions) in polyacetylene challenged the foundations of quantum physics. The study of solitons in polyacetylene (Heeger et al., 1988), stimulated by the SuSchrieffer-Heeger (SSH) papers (Su et al., 1979, 1980), dominated the first half of the 1980s.

The opportunity to synthesize new conducting polymers with improved/desired properties began to attract the attention of synthetic chemists in the 1980s. Although it would be an overstatement to claim that chemists can now control the energy gap of semiconducting polymers through molecular design, we certainly have come a long way toward that goal.

Reversible "doping" of conducting polymers, with associated control of the electrical conductivity over the full range from insulator to metal, can be accomplished either by chemical doping or by electrochemical doping. Concurrent with the doping, the electrochemical potential (the Fermi level) is moved either by a redox reaction or an acid-base reaction into a region of energy where there is a high density of electronic states; charge neutrality is maintained by the introduction of counterions. Metallic polymers are, therefore, salts. The electrical conductivity results from the existence of charge carriers (through doping) and from the ability of those charge carriers to move along the $\pi$-bonded "highway." Consequently, doped conjugated polymers are good conductors for two reasons:

Doping introduces carriers into the electronic structure. Since every repeat unit is a potential redox site, conjugated polymers can be doped $n$ type (reduced) or $p$ type (oxidized) to a relatively high density of charge carriers (Chiang et al., 1978).

(ii) The attraction of an electron in one repeat unit to the nuclei in the neighboring units leads to carrier delocalization along the polymer chain and to charge-carrier mobility, which is extended into three dimensions through interchain electron transfer.

Disorder, however, limits the carrier mobility and, in the metallic state, limits the electrical conductivity. Indeed, research directed toward conjugated polymers with improved structural order and hence higher mobility is a focus of current activity in the field.

Figure 2 shows the early results on the conductivity of polyacetylene as a function of the doping level; even in these early studies the conductivity increased by more than a factor of $10^{7}$ to a level approaching that of a metal (Chiang et al., 1977).

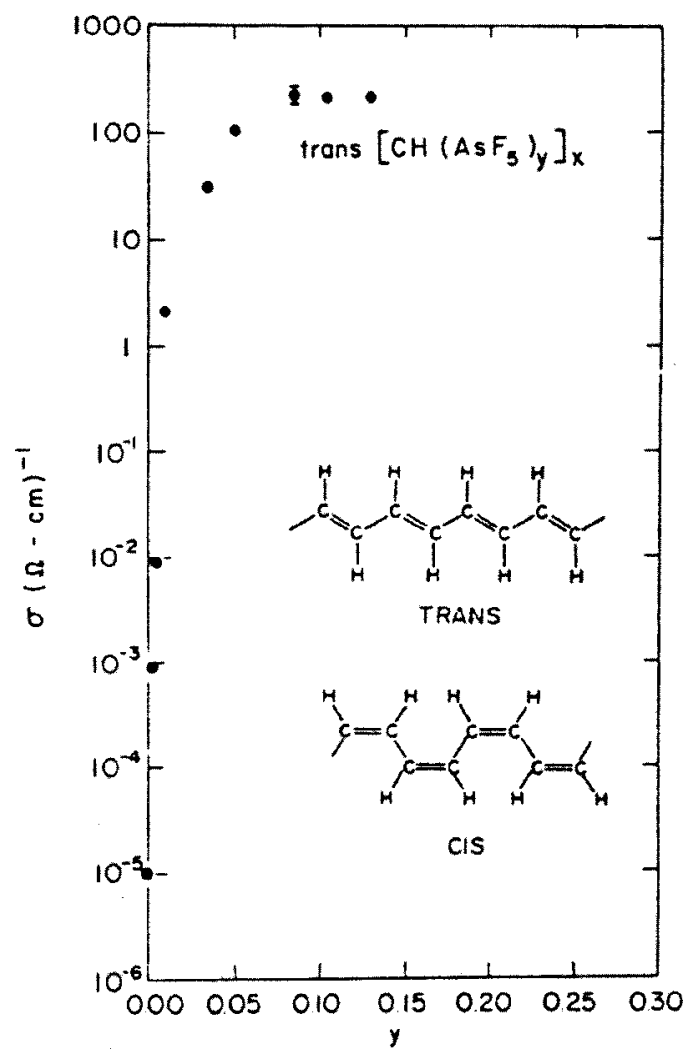

FIG. 2. Electrical conductivity of trans- $(\mathrm{CH})$, as a function of $\left(\mathrm{AsF}_{5}\right)$ dopant concentration. The trans and cis polymer structures are shown in the inset.

The electrochemical doping of conducting polymers was discovered by the MacDiarmid-Heeger collaboration in 1980 and opened the way to research in yet another scientific direction (Nigrey et al., 1979). The electrochemistry of conducting polymers has developed into a field of its own with applications that range from polymer batteries and electrochromic windows to lightemitting electrochemical cells (Pei et al., 1995).

Although I have emphasized the processing advantages of polymers, even as late as 1990 there were no known examples of stable metallic polymers which could be processed in the metallic form (a requirement for broad use in industrial products). This major outstanding problem was first solved with polyaniline, PANI. PANI has been investigated extensively for over 100 years and attracted interest as a conducting material for several important reasons: the monomer is inexpensive, the polymerization reaction is straightforward and proceeds with high yield, and PANI has excellent stability. As shown by Alan MacDiarmid and his collaborators in the mid 1980s, polyaniline can be rendered conducting through two independent routes, oxidation (either chemically or electrochemically) of the leucoemeraldine base or protonation of the emeraldine base through acid-base chemistry (Salaneck et al., 1986). Because the insertion of counterions is involved in both routes, conducting polyaniline is a salt (a polycation with one anion per repeat unit).

Processing high-molecular-weight polyaniline into useful objects and devices proved to be a difficult prob- 
lem. Yong Cao, Paul Smith, and I made important progress in 1991 by using functionalized protonic acids to both convert PANI to the metallic form and, simultaneously, render the resulting PANI complex soluble in common organic solvents (Cao et al., 1992). The functionalized counterion acts like a "surfactant" in that the charged head group is ionically bound to the oppositely charged protonated PANI chain, and the "tail" is chosen to be compatible with nonpolar or weakly polar organic liquids (in the case of solutions) or the host polymer (in the case of blends). The processibility of PANI induced by the "surfactant" counterions has made possible the fabrication of conducting polymer blends with a variety of host polymers (Cao et al., U.S. patent 5,232,631). Since the blends are melt-processible as well, the counterion-induced processibility of polyaniline provides a route to conducting polymer blends for use in industrial products. The "surfactant" counterions offer an unexpected advantage: they lead to the formation of a self-assembled network morphology in the PANI polyblends (Yang et al., 1992). Because of these interpenetrating networks, the threshold for the onset of electrical conductivity in blends with traditional insulating host polymers is reduced to volume fractions well below 1\% (Reghu et al., 1993). The PANI network is sufficiently robust that it remains connected and conducting even after the removal of the host polymer, thus opening the way to research in another new direction, the fabrication of novel electrodes for use in electronic devices. The use of "surfactant" counterions was introduced with the goal of making PANI processible in the conducting form. The self-assembly of phase-separated networks was an unexpected-but very welcomebonus.

Conducting polymers were initially attractive because of the fundamental interest in the doping and the doping-induced metal-insulator transition. However, the chemistry and physics of these polymers in their nondoped semiconducting state are of great interest because they provide a route to "plastic electronic" devices. Although polymer diodes were fabricated and characterized in the 1980s, (Tomazawa et al., 1987, 1989), the discovery of light-emitting diodes (LED's) by Richard Friend and colleagues at Cambridge in 1990 (Burroughes et al., 1990) provided the stimulus for a major push in this direction. The polymer light-emitting diode is, however, only one of a larger class of devices in the emerging class of "plastic" optoelectronic devices, including lasers, high-sensitivity plastic photodiodes (and photodiode arrays) and photovoltaic cells, ultrafast image processors (optical computers), thin-film transistors, and all-polymer integrated circuits; in each case these sophisticated electronic components are fabricated from semiconducting and metallic polymers. All of these have a common structure: They are thin-film devices in which the active layers are fabricated by casting the semiconducting and/or metallic polymers from solution (e.g., spin casting or ink-jet printing).

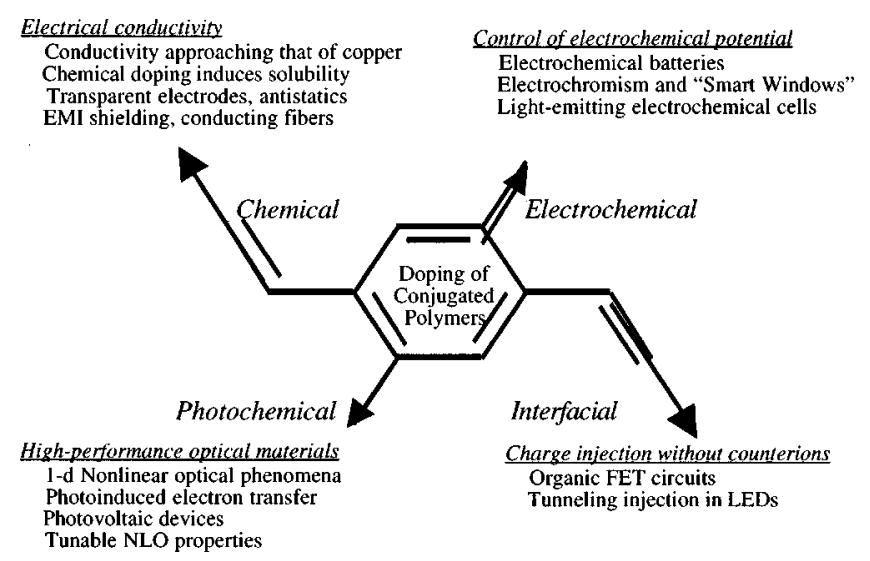

FIG. 3. Doping mechanisms and related applications.

\section{DOPING}

Charge injection onto conjugated, semiconducting macromolecular chains, "doping," leads to the wide variety of interesting and important phenomena which define the field. As summarized in Fig. 3, reversible charge injection by "doping" can be accomplished in a number of ways.

\section{A. Chemical doping by change transfer}

The initial discovery of the ability to dope conjugated polymers involved charge-transfer redox chemistry: oxidation ( $p$-type doping) or reduction ( $n$-type doping), (Chiang et al., 1977, 1978; Shirakawa et al., 1977), as illustrated with the following examples:

(a) $p$ type

$$
(\pi \text {-polymer })_{n}+\frac{3}{2} n y\left(\mathrm{I}_{2}\right) \rightarrow\left[(\pi \text {-polymer })^{+y}\left(\mathrm{I}_{3}{ }^{-}\right)_{y}\right]_{n} ;
$$

(b) $n$ type

$$
\begin{aligned}
& (\pi \text {-polymer })_{n}+\left[\mathrm{Na}^{+}(\text {Napthtalide })^{*}\right]_{y} \\
& \quad \rightarrow\left[\left(\mathrm{Na}^{+}\right)_{y}(\pi \text {-polymer })^{-y}\right]_{n}+(\text { Naphth })^{0} .
\end{aligned}
$$

When the doping level is sufficiently high, the electronic structure evolves to that of a metal.

\section{B. Electrochemical doping}

Although chemical (charge-transfer) doping is an efficient and straightforward process, it is typically difficult to control. Complete doping to the highest concentrations yields reasonably high quality materials. However, attempts to obtain intermediate doping levels often result in inhomogeneous doping. Electrochemical doping was invented to solve this problem (Nigrey et al., 1979). In electrochemical doping, the electrode supplies the redox charge to the conducting polymer, while ions diffuse into (or out of) the polymer structure from the nearby electrolyte to compensate the electronic charge. The doping level is determined by the voltage between the conducting polymer and the counterelectrode; at electrochemical equilibrium the doping level is precisely de- 


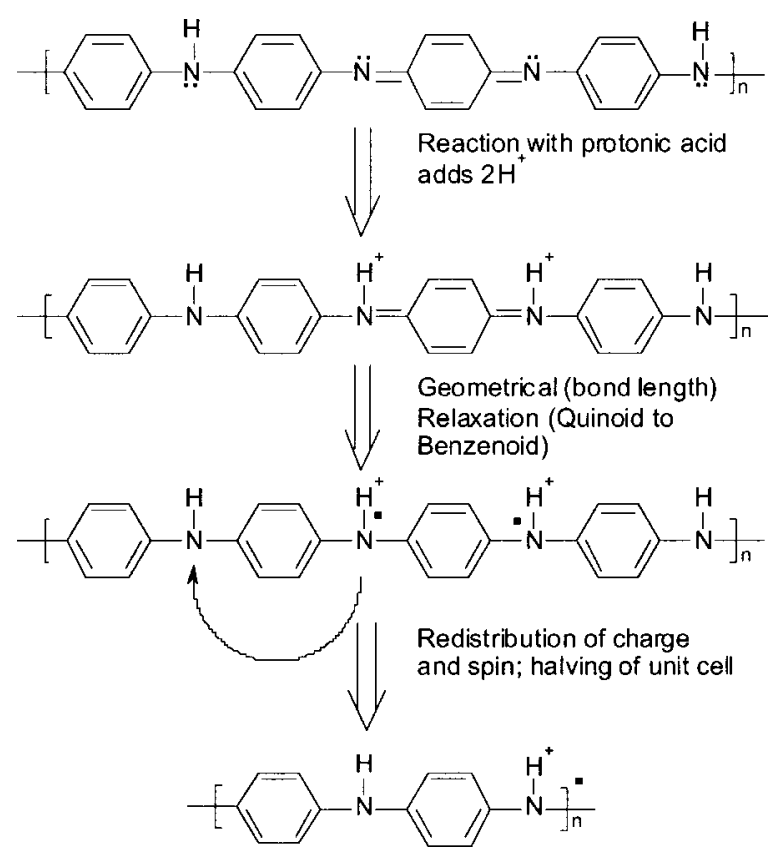

FIG. 4. Protonation-induced spin unpairing in polyaniline; conversion from insulator to metal with no change in the number of electrons.

fined by that voltage. Thus doping at any level can be achieved by setting the electrochemical cell at a fixed applied voltage and simply waiting as long as necessary for the system to come to electrochemical equilibrium (as indicated by the current through the cell going to zero). Electrochemical doping is illustrated by the following examples:

$$
\begin{aligned}
& \text { (a) } p \text { type } \\
& \qquad \begin{array}{l}
(\pi \text {-polymer })_{n}+\left[\mathrm{Li}^{+}\left(\mathrm{BF}_{4}{ }^{-}\right)\right]_{\mathrm{sol} n} \\
\quad \rightarrow\left[(\pi \text {-polymer })^{+y}\left(\mathrm{BF}_{+}{ }^{-}\right)_{y}\right]_{n}+\mathrm{Li}(\text { electrode })
\end{array}
\end{aligned}
$$

(b) $n$ type

$$
\begin{aligned}
& (\pi \text {-polymer })^{n}+\mathrm{Li}(\text { electrode }) \\
& \quad \rightarrow\left[\left(\mathrm{Li}^{+}\right)_{y}(\pi \text {-polymer })^{-y}\right]_{n}+\left[\mathrm{Li}^{+}\left(\mathrm{BF}_{4}^{-}\right)\right]_{\text {soln }} .
\end{aligned}
$$

\section{Doping of polyaniline by acid-base chemistry}

Polyaniline provides the prototypical example of a chemically distinct doping mechanism (Salaneck et al., 1986). Protonation by acid-base chemistry leads to an internal redox reaction and the conversion from semiconductor (the emeraldine base) to metal (the emeraldine salt). The doping mechanism is shown schematically in Fig. 4. The chemical structure of the semiconducting emeraldine base form of polyaniline is that of an alternating copolymer. Upon protonation of the emeraldine base to the emeraldine salt, the protoninduced spin unpairing mechanism leads to a structural change with one unpaired spin per repeat unit, but with no change in the number of electrons (Wudl et al., 1987;
MacDiarmid and Epstein, 1990). The result is a halffilled band and, potentially, a metallic state in which there is a positive charge in each repeat unit (from protonation) and an associated counterion (e.g., $\mathrm{Cl}^{-}$, $\mathrm{HSO}_{4}{ }^{-}$, DBSA ${ }^{-}$, etc.: the counterion is not shown in Fig. 4). This remarkable conversion from semiconductor to metal has been well described, but it is not well understood from the view of basic theory. There are no calculations that show that the metallic (emeraldine salt) final state is lower in energy than the semiconductor and no detailed understanding of the rearrangement reactions sketched in Fig. 4.

\section{Photodoping}

The semiconducting polymer is locally oxidized and (nearby) reduced by photoabsorption and charge separation (electron-hole pair creation and separation into "free" carriers):

$$
\begin{aligned}
&(\pi \text {-polymer })_{n}+h \nu \rightarrow {\left[\{\pi \text {-polymer }\}^{+y}\right.} \\
&\left.+\{\pi \text {-polymer }\}^{-y}\right]_{n},
\end{aligned}
$$

where $y$ is the number of electron-hole pairs (dependent upon the pump rate in competition with the recombination rate). The branching ratio between free carriers and bound excitons (and the closely related issue of the magnitude of the exciton binding energy) is a subject of continuing discussion (Sariciftci, 1998).

Following photoexcitation from the ground state $\left(1 A_{g}\right.$ in the notation of molecular spectroscopy) to the lowestenergy state with proper symmetry $\left(1 B_{u}\right)$, one finds that recombination to the ground state can be either radiative (luminescence) or nonradiative. Some families of conjugated polymers exhibit high-luminescence quantum efficiencies (for example, PPV and PPP and their soluble derivatives); others do not (for example, polyacetylene and the polythiophenes). A number of mechanisms have been identified that lead to low quantum efficiencies for photoluminescence. Rapid bond relaxation in the excited state and the formation of solitons with states at midgap prevent radiative recombination in polyacetylene (Heeger et al., 1988). The existence of an $A_{g}$ state or a triplet excited state below the $1 B_{u}$ state will favor nonradiative recombination (in both cases, direct radiative transitions to the ground state are forbidden). Interchain interactions in the excited state ("excimers") also lead to nonradiative channels for decay (Jenekhe and Osaheni, 1994; Rothberg et al., 1996; Cornil et al., 1998).

\section{E. Charge injection at a metal-semiconducting polymer (MS) interface}

Electrons and holes can be injected from metallic contacts into the $\pi^{*}$ and $\pi$ bands, respectively:

(a) Hole injection into an otherwise filled $\pi$ band

$$
\left[(\pi \text {-polymer })_{n}-y\left(e^{-}\right) \rightarrow(\pi \text {-polymer })^{+y}\right] ;
$$

(b) Electron injection into an empty $\pi^{*}$ band 


$$
\left[(\pi \text {-polymer })_{n}+y\left(e^{-}\right) \rightarrow(\pi \text {-polymer })^{-y}\right]_{n} .
$$

In the case of charge injection at an MS interface, the polymer is oxidized or reduced (electrons are added to the $\pi^{*}$ band or removed from the $\pi$ band). However, the polymer is not doped in the sense of chemical or electrochemical doping, for there are no counterions. This distinction becomes particularly clear when comparing charge injection in the polymer light-emitting diode (where there are no ions; Burroughes et al., 1990; Parker, 1994) with that in the polymer light-emitting electrochemical cell, where electrochemical doping with associated redistribution of ions provides the mechanism for charge injection (Nigrey et al., 1979).

As indicated in Fig. 3, each of the methods of chargeinjection doping leads to unique and important phenomena. In the case of chemical and/or electrochemical doping, the induced electrical conductivity is permanent, until the carriers are chemically compensated or until the carriers are purposely removed by "undoping." In the case of photoexcitation, the photoconductivity is transient and lasts only until the excitations are either trapped or decay back to the ground state. In the case of charge injection at a metal-semiconductor interface, electrons reside in the $\pi^{*}$ band and/or holes reside in the $\pi$ band only as long as the biasing voltage is applied.

Because of the self-localization associated with the formation of solitons, polarons, and bipolarons, charge injection leads to the formation of localized structural distortions and electronic states in the energy gap (Heeger et al., 1988). In the case of "photodoping," the redistribution of oscillator strength associated with subgap infrared absorption and the corresponding bleaching of the interband $\left(\pi-\pi^{*}\right)$ transition provide a route to nonlinear optical (NLO) response. Real occupation of lowenergy excited states (Heeger et al., 1988; Maniloff et al., 1997) and virtual occupation of higher-energy excited states (in the context of perturbation theory; Chiang et al., 1992) lead to, respectively, resonant and nonresonant NLO response.

By charge-injection doping at an MS interface, one can use the polymer semiconductor as the active element in thin-film diodes (Tomozawa et al., 1987, 1989) and field-effect transistors (FET's) (Burroughes et al., 1988; Garnier et al., 1994; Drury et al., 1998). Tomozawa et al. $(1987,1989)$ demonstrated the first example of an electronic device component fabricated by casting the active polymer directly from solution; even these early diodes exhibited excellent current-voltage characteristics. Dual carrier injection in metal/polymer/metal structures provides the basis for polymer light-emitting diodes (Parker, 1994). In polymer LED's, electrons and holes are injected from the cathode and anode, respectively, into the undoped semiconducting polymer; light is emitted when the injected electrons and holes meet in the bulk of the polymer and recombine with the emission of radiation (Burroughes et al., 1990).

Thus, as summarized in Fig. 3, doping is a common feature of conducting polymers; doping leads to a remarkably wide range of electronic phenomena.

\section{NOVEL PROPERTIES GENERATE NEW TECHNOLOGY}

As emphasized in the Introduction, conducting polymers exhibit novel properties not typically available in other materials. I focus on a few of these as illustrative examples:

(a) Semiconducting and metallic polymers that are soluble in and processible from common solvents;

(b) Transparent conductors;

(c) Semiconductors in which the Fermi energy can be controlled and shifted over a relatively wide range.

In each case, the property is related to a fundamental feature of the chemistry and/or physics of the class of conducting polymers. These novel properties make possible a number of applications including polymer LED's, conducting polymers as electrochromic materials, polymer photodetectors, and polymer photovoltaic cells.

\section{A. Semiconducting and metallic polymers that are soluble in and processible from common solvents}

Because the interchain electron transfer interactions of conjugated polymers are relatively strong compared with the van der Waals and hydrogen bonding interchain interactions typical of saturated polymers, conducting polymers tend to be insoluble and infusible. Thus there was serious doubt in the early years following the discovery that $\pi$-conjugated polymers could be doped to the metallic state as to whether or not processing methods could be developed. Significant progress has been made using four basic approaches:

(1) Side-chain functionalization, principally used for processing semiconducting polymers from solution in organic solvents or from water;

(2) Precursor route chemistry, principally used for processing polyacetylene and poly(phenylene vinylene) (PPV) into thin films;

(3) Counterion-induced processing, principally used for processing polyaniline in the metallic form from organic solvents;

(4) Aqueous colloidal dispersions created by template synthesis, principally used for processing polyaniline and poly(ethylenedioxythiophene), PEDOT.

The addition of moderately long side chains onto the monomer units resulted in derivatives of polythiophene, the poly(3-alkylthiophenes), or P3AT's, see Fig. 1. Since the side chains decrease the interchain coupling and increase the entropy, these derivatives can be processed either from solution or from the melt. Similarly, sidechain functionalization of poly(phenylene vinylene), PPV (see Fig. 1), has progressed to the point where a variety of semiconducting polymers and copolymers are available with energy gaps that span the visible spectrum (Hide et al., 1996). Water solubility was achieved by incorporating polar groups such as $\left(\mathrm{CH}_{2}\right)_{n} \mathrm{SO}_{3}{ }^{-} M^{+}$into the side chains (so-called "self-doped" polymers; Kobayashi et al., 1985; Heeger and Smith, 1991). 
The ability to fabricate optical-quality thin films by spin casting from solution has proven to be an enabling step in the development of plastic electronic devices such as diodes, photodiodes, LED's, light-emitting electrochemical cells (LEC's), optocouplers, and thin-film transistors (Yu and Heeger, 1996).

The counterion-induced processibility of "metallic" polyaniline utilizes bifunctional counterions such as dodecylbenzenesulfonate to render the polymer soluble (Cao, Smith, and Heeger, 1992). The charge on the $\mathrm{SO}_{3}{ }^{-}$ head group forms an ionic bond with the positive charge (proton) on the PANI chain; the hydrocarbon tail "likes" organic solvents. Processing PANI in the conducting form resulted in materials with improved homogeneity and crystallinity, and with correspondingly improved electrical conductivities (Menon et al., 1998). The solubility of "metallic" PANI in organic solvents has also made possible the fabrication of conducting blends of PANI with a variety of insulating host polymers (Cao, Smith, and Heeger, 1992, patent). These polymer blends exhibit a remarkably low percolation threshold as a result of the spontaneous formation of an interpenetrating network morphology (Yang et al., 1992).

Template-guided synthesis of conducting polymers was first reported by S. C. Yang and colleagues (Heywang and Jonas, 1992; Sun and Yang, 1992). The molecular template, in most cases polyacids such as polystyrene sulfonic acid, binds the monomer, for example aniline, to form molecular complexes that are dispersed in water as colloidal particles. Upon polymerization, the aniline monomers form polyaniline and remain attached to the template to form the template-polyaniline complex. By judicious choice of the template molecule and the polymerization conditions, stable submicron-size colloidal particles of polyaniline-template aggregate can be formed during polymerization. The stabilization against coagulation arises from the Coulomb repulsion between particles, which is a result of the surface charge provided by the extra sulfonic acid groups in polystyrene sulfonic acid. Very stable dispersions of polyanilinepolystyrene sulfonic acid complexes can be made with particle sizes less than $1 \mu \mathrm{m}$ (Heywang and Jonas, 1992; Sun and Yang, 1992). Transparent films with a specific resistivity of $1-10 \Omega \mathrm{cm}$ can be cast from such dispersions.

Poly(ethylenedioxythiophene)-polystyrene sulfonate (PEDOT-PSS) can be prepared as a stable dispersion in water (Heywang and Jonas, 1992; Sun and Yang, 1992; Friedrich and Werner, U.S. patent 5,300,575). Films of PEDOT-PSS are semitransparent and can be spin cast with a surface resistance of approximately $500 \Omega$ /square and with $75 \%$ transmission.

\section{B. Transparent metallic polymers}

Metals reflect light at frequencies below the plasma frequency $\omega_{p}$, defined as

$$
\omega_{p}^{2}=4 \pi N e^{2} / m^{*}
$$

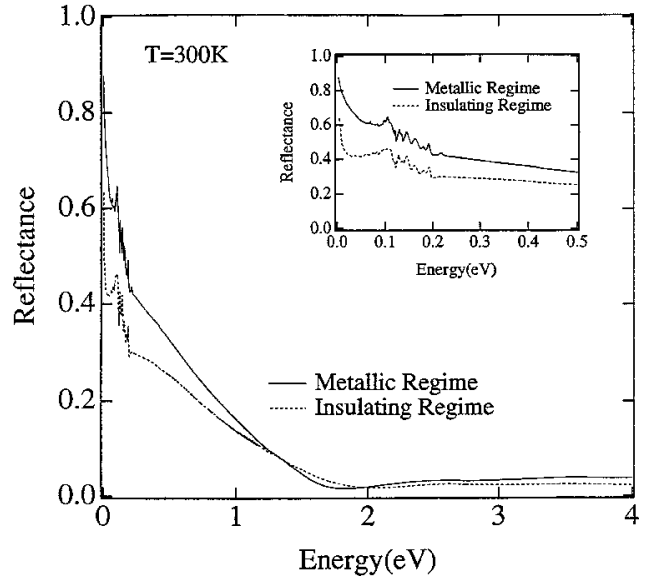

FIG. 5. Reflectance spectra of $\mathrm{PP} y-\mathrm{PF}_{6}$ near the metalinsulator transition; the inset shows the low-energy spectra with expanded scale.

where $N$ is the number of electrons per unit volume, $e$ is the electron charge, and $m^{*}$ is the effective mass (the "optical mass") of the electrons in the solid. At frequencies above the plasma frequency, metals are transparent (Kittel, 1986). For conventional metals ( $\mathrm{Na}, \mathrm{Cu}, \mathrm{Ag}$, etc.), $N$ is of order $10^{23}$ per unit volume. As a result, the plasma frequency is in the ultraviolet; therefore conventional metals appear shiny and "metallic looking" in the spectral range over which the human eye is sensitive.

Metallic polymers have a lower density of electrons; both the length of the repeat unit along the chain and the interchain spacing are relatively large compared to the interatomic distances in conventional metals. Typically therefore for metallic polymers $N$ is of order $2-5$ $\times 10^{21} \mathrm{~cm}^{-3}$. Thus, for metallic polymers, the plasma frequency is at approximately $1 \mathrm{eV}$ (Lee, Heeger, and Cao, 1993; Lee et al., 1995). The reflectance of high quality, metallic polypyrrole (doped with $\mathrm{PF}_{6}$ ) is shown in Fig. 5 . Metallic polymers exhibit high reflectance (and thus look "shiny") in the infrared, but they are semitransparent in the visible part of the spectrum. The residual absorption above the plasma frequency arises from interband $\left(\pi-\pi^{*}\right)$ transitions between the partially filled $\pi$ band and the lowest-energy $\pi^{*}$ band.

Optical-quality thin films of metallic polymers are useful, therefore, as transparent electrodes (Cao, Treacy, et al., 1992). For example, polyaniline (Gustafsson et al., 1992), polypyrrole (Gao et al., 1996), and PEDOT (Cao et al., 1997) have been used as transparent hole-injecting electrodes in polymer LED's (the initial demonstration of mechanically flexible polymer LED's utilized PANI as the anode (Gustafsson et al., 1992). Transparent conducting films can be used for a variety of purposes; for example, as antistatic coatings, as electrodes in liquidcrystal display cells or in polymer LED's, or for fabricating electrochromic windows.

\section{Semiconductors in which the Fermi energy can be shifted across the energy gap}

In comparison with traditional inorganic semiconductors, semiconducting polymers cannot be considered ma- 
terials with ultrahigh purity. As a result, although many device concepts have been demonstrated using semiconducting polymers as the active materials (Yu and Heeger, 1996), there was early skepticism that these novel semiconductors could be used in commercial applications.

In some ways, however, semiconducting polymers are more robust than their inorganic counterparts. In particular, whereas pinning of the Fermi energy by surface states is a major problem in conventional semiconductors, the Fermi energy can be controlled and shifted all the way across the energy gap in conjugated polymers. The absence of Fermi-level pinning and the ability to shift the chemical potential all the way across the energy gap are fundamentally important; these novel features of semiconducting polymers underlie the operation of polymer LED's, polymer LEC's, and polymer photodiodes.

One might have expected chemical reactions between the metal electrode and the polymer to lead to interface states that pin the Fermi level, as in inorganic semiconductors. Experiments have shown, however, that these interfacial interactions do not lead to pinning of the Fermi level. For example, electroabsorption measurements were used to determine the built-in electric field in metal-semiconductor-metal (MSM) structures of the kind used for polymer LED's (Campbell et al., 1996). The results indicated that the maximum internal field is nearly equal to the single-particle energy gap; the built-in field directly tracked the difference in work functions of the two metal electrodes, as originally proposed by Parker (1994). Thus the Fermi level is not pinned by surface states. The absence of Fermi-level pinning in semiconducting polymers is a major advantage: Conceptually, it greatly simplifies the device physics, and technologically, it greatly simplifies the device fabrication.

\section{SEMICONDUCTING POLYMERS: ELECTRONIC STRUCTURE AND BOND RELAXATION IN EXCITED STATES}

\section{A. Band structure, electron-lattice interaction, electron- electron interaction, and disorder}

Although the linear and nonlinear optical properties of conducting polymers have been investigated for over a decade, there is still controversy over the description of the elementary excitations. Are the lowest-energy elementary excitations mobile charge carriers (charged polarons) either injected at the contacts or created directly via interband photoexcitation, or are the lowestenergy excitations bound neutral excitons (Sariciftci, 1998)? The answer is of obvious importance from the perspective of our basic understanding of the physics of conducting polymers. The answer is also important for applications based on these materials.

The central issue relates to the strength of the electron-electron interactions relative to the bandwidth, relative to the electron-phonon interaction, and relative to the strength of the mean disorder potential. Strong electron-electron interactions (electron-hole attraction) lead to the creation of localized and strongly correlated negative and positive polaron pairs: neutral polaron excitons. Well-screened electrons and holes with associated lattice distortions (charged polarons), on the other hand, are more appropriately described using a band picture supplemented by the electron-phonon interaction. Molecular solids such as anthracene (Pope and Swenberg, 1982) are examples of the former, where the absorption is dominated by excitonic features, whereas inorganic semiconductors such as $\mathrm{Si}$ and GaAs are examples of the latter, where rigid band theory is a good approximation.

The electronic structure of conjugated polymers was described by Su, Schrieffer, and Heeger $(1979,1980)$ in terms of a quasi-one-dimensional tight-binding model in which the $\pi$ electrons are coupled to distortions in the polymer backbone by the electron-phonon interaction. In the SSH model, photoexcitation across the $\pi$ - $\pi^{*}$ band gap creates the self-localized, nonlinear excitations of conducting polymers: solitons (in degenerate groundstate systems), polarons, and bipolarons (Heeger et al., 1988). Direct photogeneration of solitons and polarons is made possible by the Franck-Condon overlap between the uniform chain in the ground state and the distorted chain in the excited state (Hagler et al., 1991; Hagler and Heeger, 1992). When the ground state is nondegenerate, as in the PPV's, charged polaron pairs can either separate as mobile charged polarons or form bound polaron excitons, i.e., neutral bipolarons bound by a combination of their Coulomb attraction and their shared distortion. Photoluminescence can be described in terms of the radiative decay of polaron excitons.

Conjugated polymers are $\pi$-bonded macromolecules, molecules in which the fundamental monomer unit is repeated many, many times. Thus $N$, the Staudinger index as in $(\mathrm{CH})_{N}$, is large. Since the end points are not important when $N$ is large, the $\pi$-electron transfer integral [denoted as " $\beta$ " in molecular orbital theory (Streitweiser, 1961) and " $t$ " in tight-binding theory] tends to delocalize the electronic wave functions over the entire macromolecular chain. This tendency toward delocalization is limited by disorder (which tends to localize the wave functions) and by the Coulomb interaction, which binds electrons when transferred to a nearby repeat unit to the positive charge left behind i.e., to the "hole."

In principle, disorder can be controlled. Chainextended and chain-aligned samples can be prepared. Indeed, by utilizing the method of gel processing of blends of conjugated polymers in polyethylene (Smith et al., 1981), a high degree of structural order has been attained (Hagler et al., 1991; Hagler and Heeger, 1992). Thus, as a starting point, it is useful to consider idealized samples in which the macromolecular chains are chain extended and chain aligned.

The relative importance of the Coulomb interaction versus the band structure is a classic problem of the field. In tight-binding theory, the $\pi$-electron band structure extends over a bandwidth, $W=2 z t$, where $z$ is the 


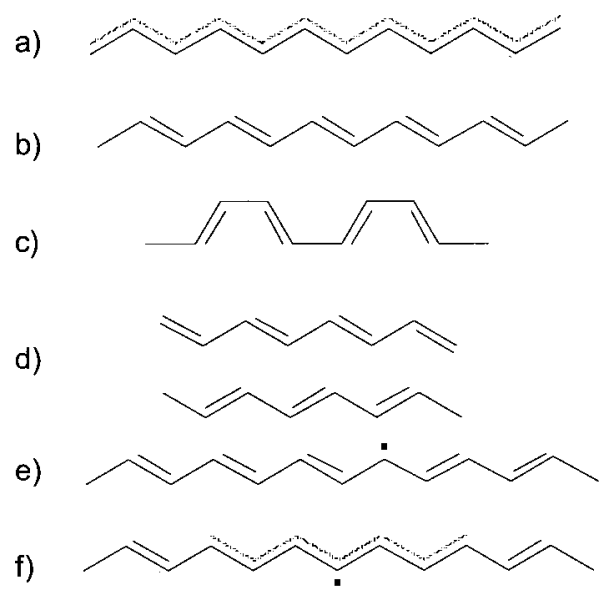

FIG. 6. Polyacetylene: (a) undimerized structure; (b) dimerized structure due to the Peierls instability; (c) cispolyacetylene; (d) degenerate $A$ and $B$ phases in transpolyacetylene; (e) soliton in trans-polyacetylene; (f) structural relaxation in vicinity of domain boundary.

number of nearest neighbors. Thus, for linear polymers with $z=2$ and $t \approx 2.5 \mathrm{eV}, W \approx 10 \mathrm{eV}$ (Heeger et al., 1988). Since the size of the monomer, typically 5-10 A along the chain axis, is the smallest length in the problem, the monomer length is the effective "Bohr radius." Thus, on general grounds, one expects the electron-hole binding energy to be reduced from $13.5 \mathrm{eV}$ (the electron-proton Coulomb binding energy in the $\mathrm{H}$ atom, where the Bohr radius is $0.53 \AA$ ) by a factor of $10-20$ simply because of the change in length scale. Dielectric screening provides an additional reduction factor of $\varepsilon$, where $\varepsilon \approx 3$ (typical of conjugated polymers) is the dielectric constant for an electric field along the chain. Thus the binding energy is expected to be no more than a few tenths of an eV. Since the typical bandwidths and band gaps are all in the $\mathrm{eV}$ range, one can start with a one-electron band approach and treat the Coulomb energy as a perturbation. In this description, the electron-hole bound states are Wannier excitons which are delocalized over a number of repeat units. There are obvious cases where this argument breaks down. For example, one finds that in structures containing benzene rings, specific subbands have bandwidths near zero (nodes in the wave function reduce the effective transfer integral to zero). When electrons and holes occupy such narrow bands, the corresponding excitons are easily localized by the Coulomb interaction onto a single monomer. Thus, in general, one can expect both "Wannier-like" excitons and "Frenkel-like" excitons for electrons and holes originating from different bands in the same polymer.

\section{B. Electronic structure of polyacetylene}

Trans-polyacetylene, trans- $(\mathrm{CH})_{x}$, was the first highly conducting organic polymer (Shivakawa et al., 1977; Chiang et al., 1977). The simple molecular structure, - $\mathrm{CH}-$ units repeated [see Fig. 6(a)], implies that each carbon contributes a single $p_{z}$ electron to the $\pi$ band. As a result, the $\pi$ band would be half filled. Thus, based

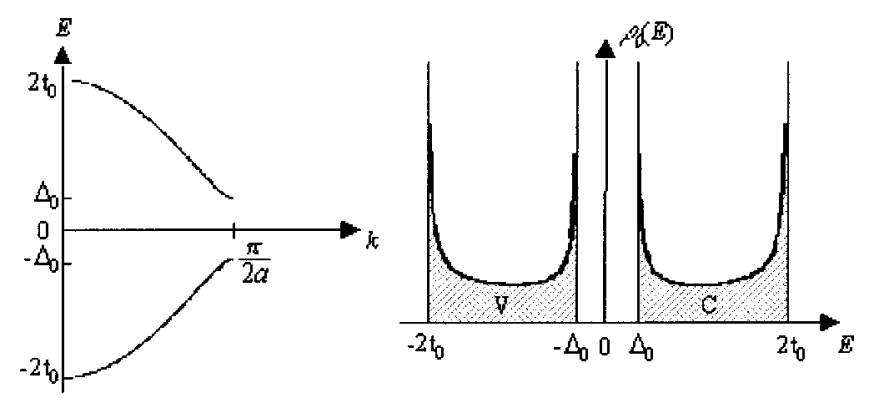

FIG. 7. Electronic structure of semiconducting polyacetylene: left, band structure; right, density of states. The energy opens at $k=\pi / 2 a$ as a result of the Peierls distortion.

upon this structure, an individual chain of neutral polyacetylene would be a metal; since the electrons in this idealized metal could move only along the chain, polyacetylene would be a metal; since the electrons in this idealized metal could move only along the chain, polyacetylene would be a one-dimensional (1D) metal. However, experimental studies show clearly that neutral polyacetylene is a semiconductor with an energy gap of approximately $1.5 \mathrm{eV}$. Rudolf Peierls (1955) showed many years ago that $1 \mathrm{D}$ metals are unstable with respect to a structural distortion which opens an energy gap at the Fermi level, thus rendering them semiconductors. In the Peierls instability, the periodicity $\left(\Lambda=\pi / k_{F}\right)$ of the distortion is determined by the magnitude of the Fermi wave vector $\left(k_{F}\right)$. Since $k_{F}=\pi / 2 a$ for the half-filled band of trans- $(\mathrm{CH})_{x}$, the Peierls distortion doubles the unit cell, converting trans-polyacetylene into trans$(-\mathrm{HC}=\mathrm{CH}-)_{x}$; see Fig. 6(b) where, schematically, the dimerization is drawn as alternating single bonds and double bonds (in reality, shorter and longer bonds). The $\pi$ band of $(\mathrm{CH})_{x}$ is split into two subbands, a fully occupied $\pi$ band (the valence band in semiconductor terminology) and an empty $\pi^{*}$ band (the conduction band), each with a wide bandwidth $(\sim 5 \mathrm{eV})$ and significant dispersion. The resulting band structure and associated density of states, shown in Fig. 7, results from the opening of the band gap that originates from the doubling of the unit cell as a result of the bond alternation caused by the Peierls instability of the $1 \mathrm{D}$ metal.

Shortly after the initial discovery of doping and the metal-insulator transition in polyacetylene, the SSH description of the electronic structure was proposed $(\mathrm{Su}$ et al., 1979, 1980). The construction of the remarkably successful SSH Hamiltonian was based on two assumptions: (a) The $\pi$-electronic structure can be treated in the tight-binding approximation with a transfer integral $t \approx 2.5 \mathrm{eV}$, and (b) the chain of carbon atoms is coupled to the local electron density through the length of the chemical bonds,

$$
t_{n, n+1}=t_{0}+\alpha\left(u_{n+1}-u_{n}\right),
$$

where $t_{n, n+1}$ is the bond-length-dependent hopping integral from site $n$ to $n+1$, and $u_{n}$ is the displacement from equilibrium of the $n$th carbon atom. The first assumption defines the lowest-order hopping integral $t_{0}$ in the tight-binding term that forms the basis of the Hamil- 


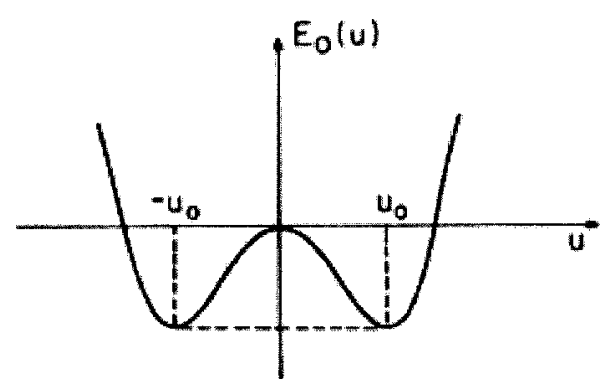

FIG. 8. Total energy of the dimerized polyacetylene chain. Note the double minimum associated with the spontaneous symmetry breaking and the twofold-degenerate ground.

tonian. The second assumption provides the first-order correction to the hopping integral. This term couples the electronic states to the molecular geometry, giving the electron-phonon interaction where $\alpha$ is the electronphonon coupling constant. The bond-length-dependent hopping integral is physically correct, as indicated by bond alternation observed in polyacetylene (Fincher et al., 1982; Yannoni and Clarke, 1983). The precise form of Eq. (8), in which the dependence of the hopping integral on the C-C distance is linearized for small deviations about $t_{0}$, is the first term in a Taylor expansion. The resulting SSH Hamiltonian is then written as the sum of three terms (Su et al., 1979, 1980):

$$
\begin{aligned}
H_{\mathrm{SSH}}= & \sum_{n, \sigma}\left[-t_{0}+\alpha\left(u_{n+1}-u_{n}\right)\right]\left(c_{n+1, \sigma}^{+} c_{n, \sigma}+c_{n, \sigma}^{+} c_{n+1, \sigma}\right) \\
& +\sum_{n}\left[\frac{p_{n}^{2}}{2 m}+\frac{1}{2} K_{n}\left(u_{n+1}-u_{n}\right)^{2}\right]
\end{aligned}
$$

where $p_{n}$ are the nuclear momenta, $u_{n}$ are the displacements from equilibrium, $m$ is the carbon mass, and $K$ is an effective spring constant. The $c_{n, \sigma}^{+}$and $c_{n, \sigma}$ are the fermion creation and annihilation operators for site $n$ and spin $\sigma$. The last two terms are, respectively, a harmonic "spring constant" term which represents the increase in potential energy that results from displacement from the uniform bond lengths in $(\mathrm{CH})_{x}$ and a kineticenergy term for the nuclear motion.

The spontaneous symmetry breaking that results from the Peierls instability implies that for the ground state of a pristine chain, the total energy is minimized for $\left|u_{n}\right|>0$. Thus, to describe the bond alternation in the ground state,

$$
u_{n} \rightarrow\left\langle u_{n}\right\rangle=(-1)^{n} u_{0} .
$$

With this mean-field approximation, the value $u_{0}$ which minimizes the energy of the system can be calculated as a function of the other parameters in the Hamiltonian (Su et al., 1979, 1980; Heeger et al., 1988). Qualitatively, however, one sees that $u_{0}$ and $-u_{0}$ both minimize the energy for trans-polyacetylene, since the bonds all make the same angle with respect to the chain axis. Hence the energy as a function of $u$ has a double minimum at $\pm u_{0}$, as shown in Fig. 8. The corresponding structures of the two degenerate ground states are shown in Fig. 6(d). The double minimum of Fig. 8 implies that nonlinear excitations, solitons, will be important. In polymers with a nondegenerate ground state, the degeneracy indicated

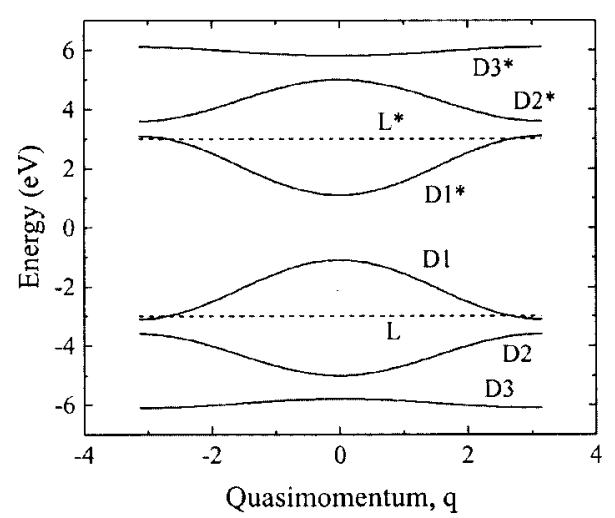

FIG. 9. Electronic band structure of PPV.

in Fig. 8 is lifted; for a nondegenerate ground-state polymer, the absolute minimum energy occurs at a single value of $u$.

\section{The electronic structure of poly(phenylene vinylene)}

Poly(phenylene vinylene), PPV, and its soluble derivatives have emerged as the prototypical luminescent semiconducting polymers. Since PPV has a nondegenerate ground state, structural relaxation in the excited state leads to the formation of polarons, bipolarons, and neutral excitons. Prior to treating the structural relaxation in the excited state, however, one needs to develop a satisfactory description of the electronic excited states.

In PPV, with eight carbons in the main-chain repeat unit, the $\pi$ band is split into eight subbands. Since each band can hold precisely two electrons per repeat unit, the four $\pi$ subbands with the lowest energy are filled and the four $\pi^{*}$ subbands are empty. Thus I begin with a description at the one-electron level, i.e., from the point of view of band theory.

Brazovskii, Kirova, and Bishop (Brazovskii et al., 1998; Kirova et al., 1999) treated the band structure of PPV with a tight-binding Hamiltonian, using standard values for the hopping integrals. The electron-phonon coupling, Eq. (8), can be included after defining the basic band structure of the semiconductor. From this simple ansatz, basis states that reflect the intrinsic symmetry of the phenyl ring and the dimer were calculated and used to expand the wave function. The basis states hybridize as a result of ring-to-dimer hopping, thus yielding the $6+2=8$ subbands in the $\pi$ system of PPV.

The resulting band structure, Fig. 9, has a number of important features. Six of the subbands (three occupied and three unoccupied), labeled $D$ and $D^{*}$ in Fig. 9, are broad, and the corresponding wave functions are delocalized along the chain. The other two bands, labeled $L$ and $L^{*}$ (one occupied and one unoccupied), are narrow, and the corresponding wave functions have amplitudes that are localized on the ring. The $L$ and $L^{*}$ subbands derive from the two ring states whose wave functions have nodes at the para linkage sites; as a result, these states do not participate in hopping and the subbands do not acquire the resulting dispersion. On the contrary, the 
delocalized $D$ and $D^{*}$ subbands have relatively high dispersion $(\sim 1.7 \mathrm{eV})$, indicating good delocalization over both ring and dimer states.

Starting from this simple yet physically robust model of the band structure, the discussion of the electronic structure can be extended to include the effect of the Coulomb interaction. The delocalized nature of $D 1$ and $D 1 *$ implies that, for electron correlation effects involving these subbands, it is appropriate to compute an effective mass from the dispersion curves and then compute the corresponding one-dimensional (1D) hydrogenic levels. From the $k^{2}$ dependence of the dispersion near the zone center, the effective masses in $D 1$ and $D 1^{*}$ are $m^{*}=0.067 m_{e}$.

Using the well-known result for the binding energy of a hydrogeniclike state in $1 \mathrm{D}$, the exciton associated with the lowest-energy electronic transition $(D 1-D 1 *)$ has a binding energy and effective radii given by

$$
E_{b}=E_{b}^{*} \ln ^{2}\left(a_{b} / a_{\perp}\right) \quad a_{b}=a_{b}^{*}\left[\ln \left(a_{b} / a_{\perp}\right)\right]^{-1},
$$

where

$$
E_{b}^{*}=\frac{m^{*} e^{4}}{\varepsilon^{2} \hbar^{2}} \quad a_{b}^{*}=\frac{\hbar^{2} \varepsilon}{m^{*} e^{2}},
$$

$\varepsilon$ is the dielectric susceptibility, $\hbar$ is Planck's constant, $m^{*}$ is the electron effective mass at the zone center in $k$ space (for PPV, $m^{*} \approx 0.067 m_{e}$ ), $e$ is the electron charge, and $a_{\perp} \approx 2 \AA$ (the "width" of the chain). From Eq. (11), $E_{b} \approx 0.1-0.2 \mathrm{eV}$ (depending on the value assumed for $a_{\perp}$ ) and a radius of approximately $30 \AA$.

In addition to this weakly bound Wannier-Mott exciton, there are two other excitons in this model. The degenerate $D 1-L 1^{*}$ and $L 1-D 1^{*}$ transitions are treated by assuming an immobile (massive) carrier in the $L$ or $L^{*}$ subband and a mobile carrier in the $D$ or $D^{*}$ subband. The resulting bound state has a binding energy of $\sim 0.8 \mathrm{eV}$ and is a more tightly bound (but still somewhat delocalized) exciton. Finally, the $L-L^{*}$ transition forms a very tightly bound Frenkel exciton, localized on the phenyl ring.

With the electronic excitations established, they can be compared to the data obtained from optical absorption measurements. An unambiguous test of the agreement between the proposed electronic structure (Fig. 9) and experiment requires data from chain-extended and chain-oriented samples of macromolecular PPV's. With macromolecular samples, chain-end boundary conditions are not important; with oriented polymers, the polarization of the various absorptions with respect to the chain axis can be determined. Recent polarized absorption studies of highy oriented PPV and polyparaphenylene (PPP) indicate that the one-electron model is a good starting point (Miller et al., 1999, 2000).

\section{Solitons, polarons, and polaron excitons: The elementary excitations of conducting polymers}

Although bond relaxation in the excited state is implicitly allowed through the bond-length-dependent hopping integral in the SSH model, the effect of bond (a)
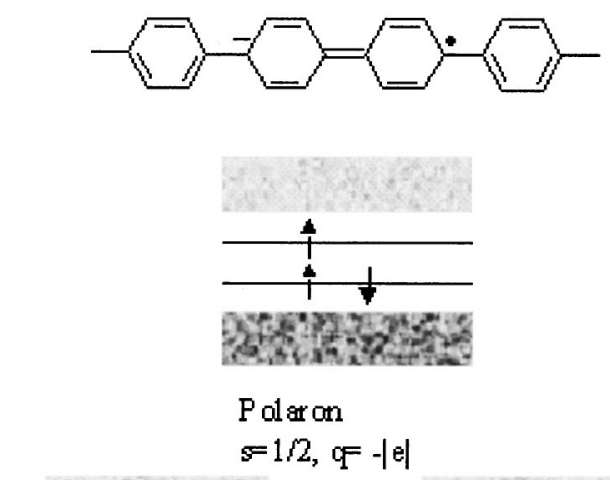

(c)

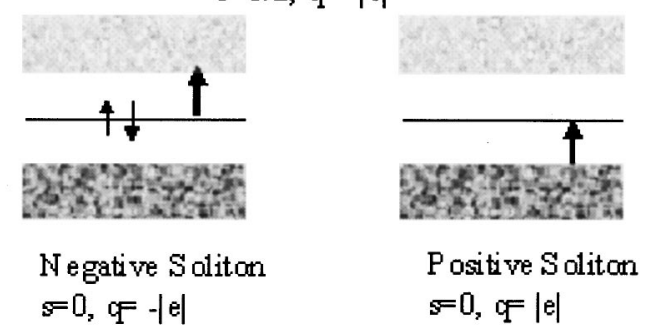

FIG. 10. Polarons in semiconducting polymers: (a) schematic picture of a polaron in PPP; (b) band diagram of an electron polaron; for a hole polaron, the lower gap state is single occupied and the upper gap state is empty; (c) band diagrams for positive and negative solitons with associated electronic transitions.

relaxation and the formation of solitons, polarons, and bipolarons have not been treated explicitly in the previous section. These important concepts are summarized in the following paragraphs. In the related experimental studies, trans-polyacetylene and polythiophene were used as the model systems for the degenerate groundstate polymer and the nondegenerate ground-state polymer, respectively (Heeger et al., 1988).

\section{Solitons}

Charge storage on the polymer chain leads to structural relaxation, which in turn localizes the charge. The simplest example of the dramatic effect of this structural relaxation is the soliton in trans-polyacetylene. The soliton is a domain boundary between the two possible degenerate ground-state configurations of trans$(-\mathrm{CH}=\mathrm{CH}-)_{N}$, the " $A$ " phase and the " $B$ " phase. For simplicity, we often draw the chemical structure of the soliton as an abrupt change from $A$ phase to $B$ phase, as shown in Fig. 6(e). In agreement with the predictions of the SSH model (Heeger et al., 1988), however, the experimental evidence indicates that the structural relaxation in the vicinity of the domain boundary extends over approximately 14 carbon atoms, as illustrated in Fig. 6(f). The corresponding spin and charge distributions are similarly delocalized.

The value of a picture like Fig. 6(e) quickly becomes evident when one tries to understand the quantum numbers of the soliton and the reversed charge-spin relationship that characterizes the solitons of polyacetylene [see Fig. 10(c)]. Since the nonbonding, or midgap, state formed by the chain relaxation can be mapped to a 
specific atomic site, the resulting distribution of charge and spin can be easily addressed; if the state is unoccupied (doubly occupied), the carbon atom at the boundary is left with a positive (negative) charge, but there are no unpaired spins; the charged soliton is positively (negatively) charged and spinless. Single occupation of the soliton state neutralizes the electronic charge of the carbon nucleus, while introducing an unpaired spin onto the chain. The localized electronic state associated with the soliton is a nonbonding state at an energy which lies at the middle of the $\pi$ - $\pi^{*}$ gap, between the bonding and antibonding levels of the perfect chain. On the other hand, the defect is both topological and mobile due to the translational symmetry of the chain. Such a topological and mobile defect is historically referred to as a soliton (Su et al., 1979, 1980; Heeger et al., 1988). The term "soliton" $(S)$ refers simultaneously to all three types of solitons; the quantum numbers for spin and charge enter only when referring to a specific type (for example, neutral solitons found as defects from the synthesis of undoped material or charged solitons created by photoexcitation), and even then, the spin is only implicit. Another feature of the soliton terminology is the natural definition of an "antisoliton" (AS) as a reverse boundary from $B$ phase back to $A$ phase in Figs. 6(e) and 6(f). The antisoliton allows for conservation of soliton number upon doping or upon photoexcitation.

\section{Polarons and bipolarons}

In cases such as poly(thiophene), PPV, PPP, and cispolyacetylene where the two possible bond alternation patterns are not energetically degenerate, confined soliton-antisoliton pairs, polarons, and bipolarons, are the stable nonlinear excitation and the stable charge storage states (Brazovskii and Kirova, 1981; Fesser et al., 1983). A polaron can be thought of as a bound state of a charged soliton and a neutral soliton whose midgap energy states hybridize to form bonding and antibonding levels. The neutral soliton contributes no charge and a single spin, as noted above, and the charged soliton carries a charge of $\pm e$ and no spin; the resulting polaron then has the usual charge-spin relationship of fermions: $q= \pm e$ and $s=\frac{1}{2}$. The polaron is illustrated schematically for PPP in Figs. 10(a) and (b). The positive polaron is a radical cation, the negative polaron a radical anion; both are quasiparticles consisting of a single electronic charge dressed with a local geometrical relaxation of the bond lengths. Similarly, a bipolaron is a bound state of two charged solitons of like charge (or two polarons whose neutral solitons annihilate each other) with two corresponding midgap levels, as illustrated in Figs. 11(a) and (b). Since each charged soliton carries a single electronic charge and no spin, the bipolaron has charge $\pm 2 e$ and zero spin. The positive bipolaron is a spinless dication, the negative bipolaron a spinless dianion: both are doubly charged bound states of two polarons bound together by the overlap of a common lattice distortion or enhanced geometrical relaxation of the bond lengths. (a)

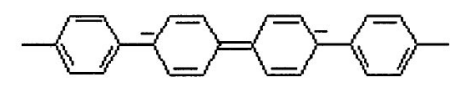

(b)

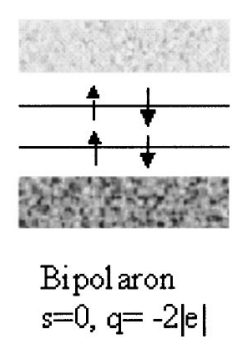

FIG. 11. Bipolarons in polymer with nondegenerate ground state: (a) schematic picture of a negative bipolaron in PPP; (b) band diagram for a negative bipolaron. For a positive bipolaron, both gap states are unoccupied.

\section{Solitons and polarons in conjugated polymers: Experimental Results}

The models outlined above for solitons, polarons, and bipolarons make concrete predictions of experimentally observable phenomena, and indeed theoretical progress would not have been possible without concurrent experimental investigation. Optical probes of the midgap electronic states [see Fig. 10(c)] and magnetic measurements of spin concentrations and spin distributions contributed greatly to the refinement and verification of theoretical predictions. For a detailed review of the experimental results, the reader is referred to the Reviews of Modern Physics article on this subject (Heeger et al., 1988).

\section{PHOTOINDUCED ELECTRON TRANSFER}

The discovery of photoinduced electron transfer in composites of conducting polymers (as donors, $D$ ) and buckminsterfullerene, $\mathrm{C}_{60}$, and its derivatives (as acceptors, $A$ ) opened a number of new opportunities for semiconducting polymers (Morita et al., 1992; Sariciftci et al., 1992). A schematic description of the photoinduced electron transfer process is displayed in Fig. 12.

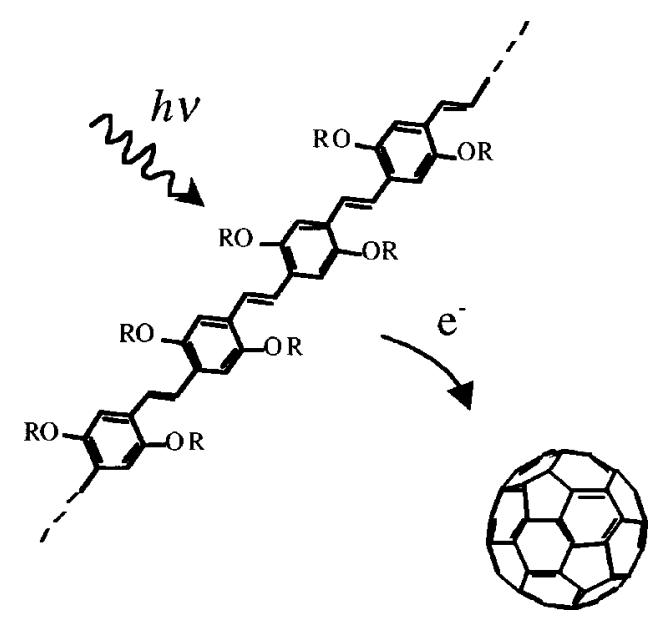

FIG. 12. Photoinduced electron transfer from a conjugated semiconducting polymer to $\mathrm{C}_{60}$. 


\section{Photoind uced Electron Transfer}

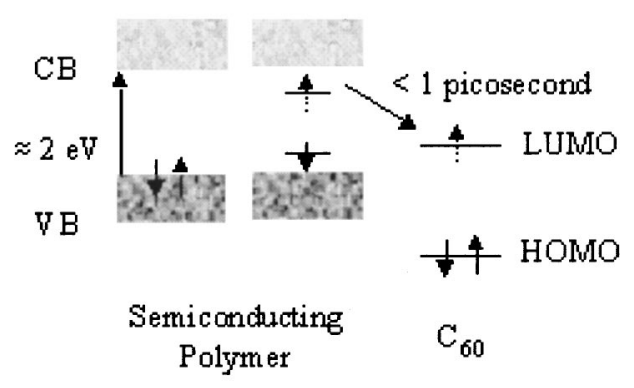

Photoinduced Hole Transfer

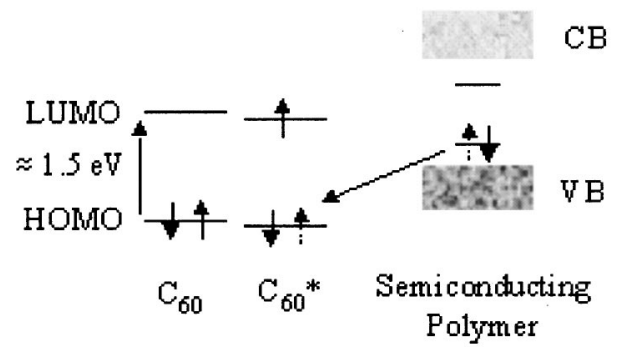

FIG. 13. Energy-band diagram for photoinduced electron transfer and photoinduced hole transfer between semiconducting polymers.

A broad range of studies has been carried out to fully characterize this photoinduced charge transfer, culminating with a study of the dynamics of photoinduced electron transfer from semiconducting polymers to $\mathrm{C}_{60}$ using fs time-resolved measurements (Kraabel et al., 1994, 1996, Lanzani et al., 2000). These studies demonstrated that the charge transfer occurs within 50 fs after photoexcitation. Since the charge transfer rate is more than 1000 times faster than any competing process (the luminescence lifetime is greater than $300 \mathrm{ps}$ ), the quantum efficiency for charge separation approaches UNITY! Moreover, the charge-transferred state is metastable (Smilowitz et al., 1993; Sariciftci and Heeger, 1994).

Semiconducting polymers are electron donors upon photoexcitation (electrons promoted to the antibonding $\pi^{*}$ band). The idea of using this property in conjunction with a molecular electron acceptor to achieve long-lived charge separation is based on the stability of the photoinduced nonlinear excitations (such as polarons) on the conjugated polymer backbone. Buckminsterfullerene, $\mathrm{C}_{60}$, is an excellent electron acceptor capable of taking on as many as six electrons (Allemand et al., 1991). It forms charge-transfer salts with a variety of strong donors. It is reasonable, therefore, to consider electron transfer from photoexcited semiconducting polymers to $\mathrm{C}_{60}$. Figure 13 shows a schematic energy-level diagram of the photoinduced electron (or hole) transfer process, which can be described in terms of the following steps:

step 1: $\quad D+A \rightarrow{ }^{1,3} D^{*}+A$ (excitation on $\left.D\right)$;

step 2: ${ }^{1,3} D^{*}+A \rightarrow{ }^{1,3}(D-A)^{*}$ (excitation delocalized on $D$ - $A$ complex);

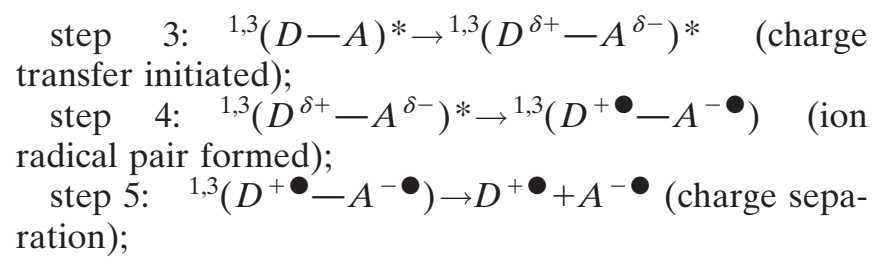

where the donor $(D)$ and acceptor $(A)$ units are either covalently bound (intramolecular) or spatially close, but not covalently bonded (intermolecular); 1 and 3 denote singlet or triplet excited states, respectively.

Since the partial charge transfer at step 3 is strongly dependent on the surrounding medium, there is a continuous range for the transfer rate, $0<\delta<1$. At step 4, $\delta=1$, i.e., a whole electron is transferred. At each step, the $D-A$ system can relax back to the ground state either by releasing energy to the "lattice" (in the form of heat) or through light emission (provided the radiative transition is allowed). The electron-transfer step (Step 4) describes the formation of an ion radical pair; this does not occur unless $I_{D^{*}}-A_{A}-U_{C}<0$, where $I_{D^{*}}$ is the ionization potential of the excited state $\left(D^{*}\right)$ of the donor, $A_{A}$ is the electron affinity of the acceptor, and $U_{C}$ is the Coulomb energy of the separated radicals (including polarization effects). Stabilization of the charge separation (Step 5) can be achieved by carrier delocalization on the $D^{+}$(and/or $\left.A^{-}\right)$species and by structural relaxation. The symmetrical process of hole transfer from the photoexcited acceptor to the donor is described in an analogous way and can be driven by photoabsorption in spectral regions where the acceptor can be photoexcited but not the donor.

Even though the photoinduced electron-transfer reaction is energetically favorable, energy must be conserved in the process. In semiconducting polymers, the excess energy is readily taken up by promoting the hole to a higher energy state in the $\pi^{*}$ band. Ultimately, therefore, the ultrafast nature of the photoinduced electrontransfer process results from the delocalized nature of the $\pi$ electrons. Once the photoexcited electron is transferred to an acceptor unit, the resulting cation radical (positive polaron) species on the conjugated polymer backbone is relatively stable. Thus photoinduced electron transfer from the conjugated polymer donor onto an acceptor moiety can be viewed as "photodoping" (see Fig. 3). The forward-to-reverse asymmetry of the photoinduced charge separation in the conducting polymer/ $\mathrm{C}_{60}$ system is nevertheless remarkable; the asymmetry is orders of magnitude greater than that observed in the photosynthesis of green plants.

Definitive evidence of charge transfer and charge separation was obtained from light-induced electron spin resonance (LESR) experiments (Sariciftci et al., 1992; Lee et al., 1994; Sariciftci and Heeger, 1994). Upon illuminating the conjugated polymer/ $\mathrm{C}_{60}$ composites with light with $h v>E_{\pi-\pi^{*}}$, where $E_{\pi-\pi^{*}}$ is the energy gap of the conjugated polymer (donor), two photoinduced electron spin resonance (ESR) signals can be resolved, one at $g=2.00$ and the other at $g=1.99$ (Sariciftci et al., 1992; Sariciftci and Heeger, 1994). The higher $g$-value line is assigned to the conjugated polymer cation 
(polaron) and the lower $g$-value line to the $\mathrm{C}_{60}{ }^{-}$anion. The assignment of the lower $g$-value line to $\mathrm{C}_{60}{ }^{-}$is unambiguous, for this low $g$ value was measured earlier (Allemand et al., 1991); the higher $g$ value is typical of conjugated polymers. The LESR signal vanishes above $200 \mathrm{~K}$; this rules out permanent photochemical changes as the origin of the ESR signal and demonstrates the reversibility of the photoinduced electron transfer. The integrated LESR intensity shows two peaks with equivalent intensities. The temperature dependence of the LESR signal intensity shows Arrhenius behavior with activation energy of approximately $15 \mathrm{meV}$. This result suggests a phonon-assisted back relaxation mechanism of the photoinduced charge-separated state (Sariciftci and Heeger, 1994).

In $\mathrm{MEH}-\mathrm{PPV} / \mathrm{C}_{60}$ composites, the strong photoluminescence of MEH-PPV (poly[2-methoxy-5( 2 '-ethyl)hexyloxy-1,4-phenylene vinylene) is quenched by a factor in excess of $10^{3}$, and the luminescence decay time is reduced from $\tau_{0}=550 \mathrm{ps}$ to $\tau_{\text {rad }} \ll 60$ ps (the instrumental resolution), indicating that charge transfer has cut off the radiative decay (Sariciftci et al., 1992; Smilowitz et al., 1993; Sariciftci and Heeger, 1994). An estimate of the transfer rate, $1 / \tau_{\mathrm{ct}}$, can be obtained from the quenching of the photoluminescence:

$$
1 / \tau_{\mathrm{ct}} \approx\left(1 / \tau_{\mathrm{rad}}\right) I_{0} / I_{\mathrm{comp}},
$$

where $1 / \tau_{0}$ and $1 / \tau_{\text {rad }}$ are the radiative decay rates, and $I_{0}$ and $I_{\text {comp }}$ are the integrated photoluminescence intensities of MEH-PPV and the MEH-PPV/C 60 composite, respectively. The data imply therefore that $1 / \tau_{\mathrm{ct}}$ $>10^{12}$; i.e., electron transfer occurs on the subpicosecond time scale. The ultrafast charge-transfer process was subsequently time resolved (Kraabel et al., 1994, 1996; Lanzani et al., 2000); the data directly confirm that charge transfer occurs within a hundred femtoseconds.

The photoinduced electron-transfer process serves to sensitize the photoconductivity of the semiconducting polymer (Lee, Yu, et al., 1993). Time-resolved transient photocurrent measurements indicate that the addition of as little as $1 \%$ of $\mathrm{C}_{60}$ into the semiconducting polymer results in an increase of initial photocurrent by an order of magnitude. This increase of the photocarrier generation efficiency is accompanied by an increase in lifetime of the photocarriers upon adding $\mathrm{C}_{60}$. Thus the ultrafast photoinduced electron transfer from the semiconducting polymer onto $\mathrm{C}_{60}$ not only enhances the charge-carrier generation in the host polymer but also serves to prevent recombination by separating the charges and stabilizing the charge separation.

Conjugated polymers with higher electron affinities (e.g., cyano-substituted PPV) function as the acceptor component, with MEH-PPV as the donor (Halls et al., 1995; Yu and Heeger, 1995). Through control of the morphology of the phase separation into an interpenetrating bicontinuous network of $D$ and $A$ phases, high interfacial area was achieved within a bulk material: a "bulk $D / A$ heterojunction" that yields efficient photoinduced charge separation (Halls et al., 1995; Yu and Hee- ger, 1995; Yu et al., 1995; Yang and Heeger, 1996). These all-polymer phase-separated blends were successfully used in fabricating solar cells with efficiencies that approach those fabricated from amorphous silicon ( $\mathrm{Yu}$ et al., 1995).

\section{METALLIC POLYMERS AND THE METAL-INSULATOR TRANSITION}

\section{A. Metallic polymers with high-performance electrical and mechanical properties}

An early (and continuing) goal of the field of conducting polymers was the creation of materials with high electrical conductivity and with the excellent mechanical properties of polymers. This goal has been achieved; more importantly, the conditions for realizing this combination of properties are understood and can be applied to new materials.

Electrical conductivity results from the existence of charge carriers and the ability of those carriers to move. In principle, broad $\pi$-electron bandwidths (often several $\mathrm{eV}$ ) can lead to relatively high carrier mobilities (Heeger et al., 1988). As a result of the same intrachain $\pi$ bonding and the relatively strong interchain electrontransfer interaction, the mechanical properties (Young's modulus and tensile strength) of conjugated polymer are potentially superior to those of saturated polymers. Thus metallic polymers offer the promise of truly high performance: high conductivity plus superior mechanical properties.

This combination of high electrical conductivity and outstanding mechanical properties has been demonstrated for doped polyacetylene (Akagi et al., 1989; Tsukamoto 1990; Cao et al., 1991; Tokito et al., 1991; Yang and Heeger, 1996; Schimmel et al., 1998).

\section{B. Metal-insulator transition in doped conducting polymers}

\section{The role of disorder}

Ioffe and Regel (1960) argued that as the extent of disorder increased in a metallic system, there was a limit to metallic behavior; when the mean free path becomes equal to the interatomic spacing, coherent metallic transport would not be possible. Thus the Ioffe-Regel criterion is defined as

$$
k_{F} l \approx 1,
$$

where $k_{F}$ is the Fermi wave number and $l$ is the mean free path. The metallic regime corresponds to $k_{F} l \gg 1$. Based on the Ioffe-Regel criterion, Mott (Mott and Davis, 1979; Mott, 1990) proposed that a metal-insulator $(M-I)$ transition must occur when the disorder is sufficiently large that $k_{F} l<1$. In recognition of Anderson's early work on disorder-induced localization, Mott called this $M-I$ transition the "Anderson transition" (Anderson, 1958). In the limit where $k_{F} l \ll 1$ (i.e., where the strength of the random disorder potential is large compared to the bandwidth), all states become localized and the system is called a Fermi glass (Anderson, 1970). A 
Fermi glass is an insulator with a continuous density of localized states occupied according to Fermi statistics. Although there is no energy gap, the behavior is that of an insulator because the states at the Fermi energy are spatially localized.

The scaling theory of localization demonstrated that the disorder-induced $M-I$ transition was a true phase transition with a well-defined critical point (Abrahams et al., 1979). McMillan (1981) and Larkin and Khmelnitskii (1982) showed that near the critical regime of Anderson localization a power-law temperature dependence is to be expected for the conductivity.

The $M-I$ transition in conducting polymers is particularly interesting; critical behavior has been observed over a relatively wide temperature range in a number of systems, including polyacetylene, polypyrrole, $\operatorname{poly}(p-$ phenylenevinylene), and polyaniline (Menon et al., 1998). In each case, the metallic, critical, and insulating regimes near the $M-I$ transition have been identified. The critical regime is tunable in conducting polymers by varying the extent of disorder (i.e., by studying samples with different $\rho_{r} \equiv \rho(1.4 \mathrm{~K}) / \rho(300 \mathrm{~K})$, or by applying external pressure and/or magnetic fields. The transitions from metallic to critical behavior and from critical to insulating behavior have been induced with a magnetic field, and those from insulating to critical and then to metallic behavior with increasing external pressure (Menon et al., 1998).

In the metallic regime, the zero-temperature conductivity remains finite with magnitude that depends on the extent of the disorder. Metallic behavior has been demonstrated for conducting polymers with $\sigma(T)$ remaining constant as $T$ approaches zero (Menon et al., 1998). Well into the metallic regime where the mean free path extends over many repeat units, the residual resistivity will become small, as in a typical metal. However, this truly metallic regime, with $k_{F} l \gg 1$, has not yet been achieved.

\section{Infrared reflectance studies of the metallic state and the metal-insulator transition}

Infrared reflectance measurements have played an important role in clarifying the metal physics of conducting polymers. High-precision reflectance measurements were carried out over a wide spectral range on a series of $\mathrm{PP}_{y}-\mathrm{PF}_{6}$ samples in the insulating, critical, and metallic regimes near the $M-I$ transition (Lee et al., 1998). Since the reflectance in the infrared (IR) is sensitive to the charge dynamics of carriers near the Fermi energy $\left(E_{F}\right)$, such a systematic reflectance study can provide information on the electronic states near $E_{F}$ and how those states evolve as the system passes through the $M-I$ transition. The data demonstrate that metallic $\mathrm{PP}_{y}-\mathrm{PF}_{6}$ is a "disordered metal" and that the $M-I$ transition is driven by disorder; similar results were obtained for polyaniline (Lee, Heeger, and Cao, 1993, 1995).

Figure 14 shows $\sigma(\omega)$ for a series of $\mathrm{PP}_{y}-\mathrm{PF}_{6}$ samples $(A-F)$ as obtained from Kramers-Kronig transformation of $R(\omega)$ at room temperature. For the mostmetallic samples, $R(\omega)$ exhibits metal-like features: a

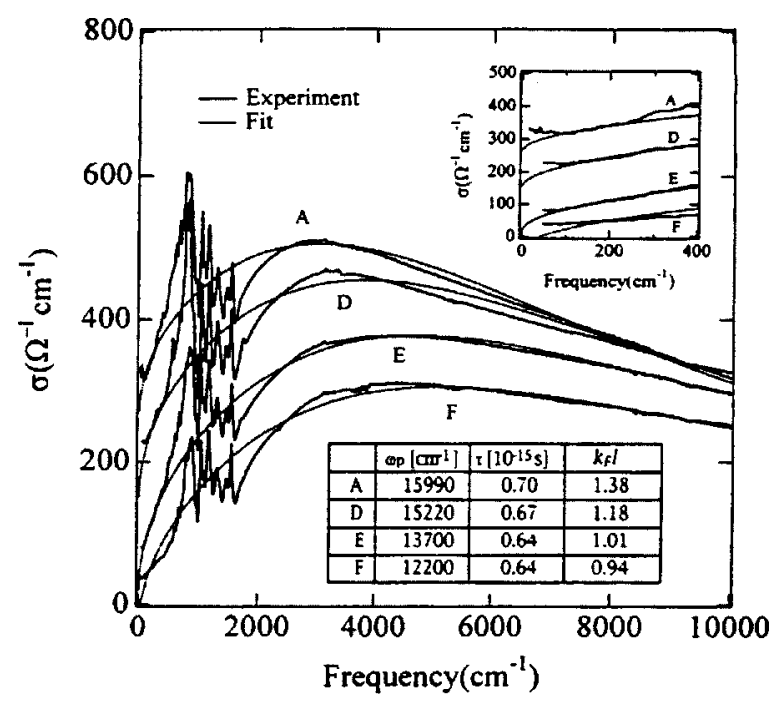

FIG. 14. Frequency-dependent conductivity $\sigma(\omega)$ for Ppy $-\mathrm{PF}_{6}$. The inset shows the far-IR data in greater detail.

free-carrier plasma resonance as indicated by a minimum in $R(\omega)$ near $1 \mathrm{eV}$ and high $R(\omega)$ in the far IR. As $\mathrm{PP}_{y}-\mathrm{PF}_{6}$ goes from the metallic to the insulating regime via the critical regime (samples $A$ through $F$ ), $\sigma(\omega)$ is gradually suppressed in the IR. In the insulating regime $(F), \sigma(\omega)$ remains well below that of the metallic sample $(A)$ throughout the IR. The $\sigma(\omega)$ spectra are in excellent correspondence with the transport results (this is especially clear at low frequencies in Fig. 14); the better the quality of the sample, as defined by the higher $\sigma_{\mathrm{dc}}(300 \mathrm{~K})$, the higher $R(\omega)$ in the IR (Lee et al., 1998).

In the far IR (below $100 \mathrm{~cm}^{-1}$ ), the Hagen-Rubens approximation provides an excellent fit to $R(\omega)$ (Kittel, 1986):

$$
R_{\mathrm{H}-\mathrm{R}}(\omega)=1-\left(2 \omega / \pi \sigma_{\mathrm{H}-\mathrm{R}}\right)^{1 / 2},
$$

where $\sigma_{\mathrm{H}-\mathrm{R}}$ is the $\omega$-independent conductivity. The $\sigma_{\mathrm{H}-\mathrm{R}}$ values obtained from the Hagen-Rubens fits are in remarkably good agreement with the measured values of $\sigma_{\mathrm{dc}}(300 \mathrm{~K})$. The excellent fits and the agreement between $\sigma_{\mathrm{H}-\mathrm{R}}$ and $\sigma_{\mathrm{dc}}(300 \mathrm{~K})$ imply a weak $\omega$ dependence in the corresponding optical conductivity, $\sigma(\omega)$, for $\omega<$ $100 \mathrm{~cm}^{-1}$.

The $\sigma(\omega)$ data are fully consistent with the "localization-modified Drude model" (LMD; Mott and Kaveh, 1985):

$$
\sigma_{\mathrm{LD}}(\omega)=\sigma_{\text {Drude }}\left\{1-C\left[1-(3 \tau \omega)^{1 / 2}\right] /\left(k_{F} l\right)^{2}\right\},
$$

where $k_{F}$ is the Fermi wave number and $l$ is the mean free path. In this model, the zero-frequency limit determines the constant $C$ (precisely at the M-I transition, $C=1)$, while a fit to $\sigma(\omega)$ determines $k_{F} l$. The suppressed $\sigma(\omega)$ as $\omega$ approaches zero arises from weak localization induced by disorder (Lee et al., 1998). The $\sigma(\omega)$ data from the various regimes were fit with the functional dependence predicted by the LMD model; Fig. 14 illustrates the excellent agreement of the fits to the data with the parameters summarized in the inset. The phonon features around $400-2000 \mathrm{~cm}^{-1}$ are, of 


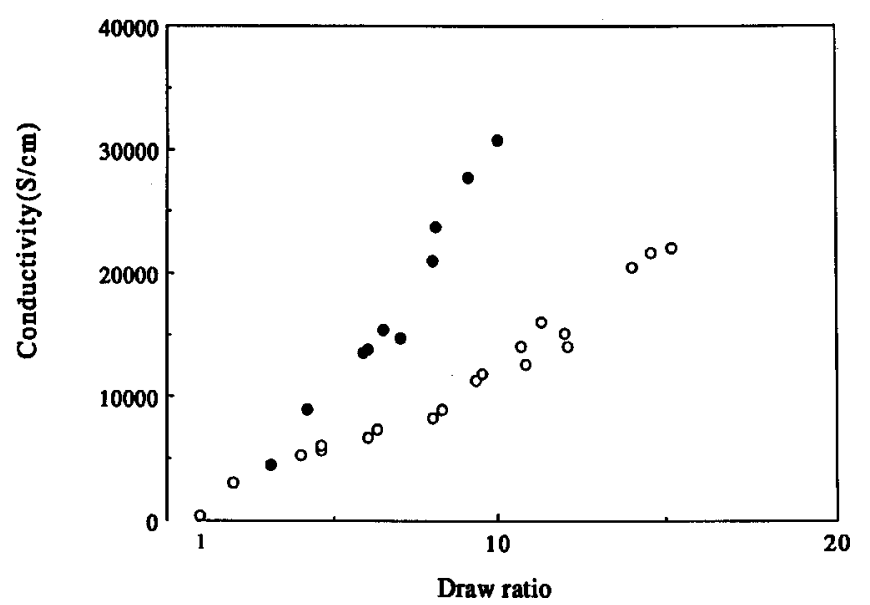

FIG. 15. Electrical conductivity of iodine-doped polyacetylene (parallel to the draw axis) vs draw ratio: solid points, thin films of thickness 3-5 $\mu \mathrm{m}$; open circles, films of thickness $25-30 \mu \mathrm{m}$.

course, not included in the LMD model. There are small deviations for $\omega<100 \mathrm{~cm}^{-1}$ (more important in the lessmetallic samples), below which phonon-assisted hopping makes a measurable contribution to $\sigma(\omega)$ and to the dc conductivity.

The parameters obtained from the fits are reasonable. The screened plasma frequency, $\Omega_{p}=\omega_{p} /\left(\varepsilon_{\infty}\right)^{1 / 2}=1.5$ $\times 10^{4} \mathrm{~cm}^{-1}$, is in good agreement with the frequency of the minimum in $R(\omega)$, and $\tau$ is typical of disordered metals $\left(\tau \sim 10^{-14}-10^{-15} \mathrm{~s}\right)$. The quantity $k_{F} l$ is of particular interest, for it characterizes the extent of disorder and is often considered as an order parameter in localization theory (Lee and Ramakrishnan, 1985; Castner, 1991). For all four samples represented in Fig. 14, $k_{F} l \approx 1$, implying that all are close to the $M-I$ transition. As the disorder increases and the system moves from the metallic regime (sample $A$ with $k_{F} l=1.38$ ) to the critical regime (sample $E$ with $\left.k_{F} l=1.01\right), k_{F} l$ approaches the Ioffe-Regel limit (Ioffe and Regel, 1960), precisely as would be expected. In the insulating regime (sample $F$ ), $k_{F} l=0.94<1$, consistent with localization of the electronic states at $E_{F}$.

Thus the IR reflectance data obtained for metallic polymers indicate that they are disordered metals near the disorder-induced $M-I$ transition. There is remarkable consistency between the conclusion obtained from transport studies and from IR reflectance measurements.

\section{Striving toward more perfect materials-chain extension, chain alignment, and interchain order}

Experimental studies have established that, for conducting polymers, the electrical properties and the mechanical properties improve together, in a correlated manner, as the degree of chain extension and chain alignment is improved. Polyacetylene remains the prototype example.

Figure 15 shows the correlation between the electrical conductivity $(\sigma)$ and the draw ratio $(\lambda)$ for iodine-doped polyacetylene films; the conductivity increases approxi- mately linearly with the draw ratio (Cao et al., 1991). The slope of $\sigma$ versus $\lambda$ is approximately a factor of 2 larger for the thinner films (evidently the details of polymerization and/or doping result in more homogeneous, higher-quality material for the thinner films).

$\mathrm{X}$-ray-diffraction studies of these drawn films exhibited a high degree of structural order that improves with the draw ratio; the structural coherence length perpendicular to the draw direction increases by about a factor of 2 as the films are drawn, from $10 \mathrm{~nm}$ at $\lambda=4$ to $20 \mathrm{~nm}$ at $\lambda=15$ (Cao et al., 1991). Consistent with the chain orientation and the improved structural order, the anisotropy $\left(\sigma_{\|} / \sigma_{\perp}\right)$ in the electrical conductivity increased with the draw ratio, approaching 250 as $\lambda \rightarrow 15$ (although $\sigma_{\|}$increased dramatically as a function of $\lambda, \sigma_{\perp}$ remains essentially constant). These data set a lower limit on the intrinsic anisotropy and demonstrate that heavily doped polyacetylene is a highly anisotropic metal with relatively weak interchain coupling.

Does the weak interchain coupling imply poor mechanical properties? The answer is "no"; for films with $\lambda=15$, Young's modulus reaches $50 \mathrm{GPa}$ and the tensile strength approaches $1 \mathrm{GPa}$, mechanical properties that are characteristic of high-performance materials. More importantly, the data demonstrate a direct correlation between the electrical conductivity and the mechanical properties. The linear relationship implies that the increases in both the conductivity and the modulus (or tensile strength) with draw ratio result from increased uniaxial orientation, improved lateral packing, and enhanced interchain interaction. The correlations between the electrical conductivity and the mechanical properties observed for polyacetylene are general. This correlation has been demonstrated in every case studied (Heeger and Smith, 1991) and can be understood as a general feature of conducting polymers.

Although the electrical conductivity is enhanced by the relatively high mobility associated with intrachain transport, one must have the possibility of interchain charge transfer to avoid the localization inherent to systems with a 1D electronic structure (Mott and Davis, 1979). The electrical conductivity becomes three dimensional (and thereby truly metallic) only if there is high probability that an electron will have diffused to a neighboring chain prior to traveling between defects on a single chain. For well-ordered crystalline material in which the chains have precise phase order, the interchain diffusion is a coherent process. In this limit, the condition for extended anisotropic transport is the following (Kivelson and Heeger, 1987):

$$
L / a \gg\left(t_{0} / t_{3 \mathrm{D}}\right) \text {, }
$$

where $L$ is the coherence length, $a$ is the length of the chain repeat unit, $t_{0}$ is the intrachain $\pi$-electron-transfer integral, and $t_{3 \mathrm{D}}$ is the interchain $\pi$-electron-transfer integral.

A precisely analogous argument can be constructed for achieving the highest possible (intrinsic) strength of a polymer material, i.e., the strength of the main-chain covalent bonds. If $E_{0}$ is the energy required to break the 
covalent main-chain bond and $E_{3 \mathrm{D}}$ is the weaker interchain bonding energy (from van der Waals forces and hydrogen bonding), then the requirement is coherence over a length $L$ such that (Heeger, 1989)

$$
L / a \gg E_{0} / E_{3 \mathrm{D}} .
$$

In this limit, the large number, $L / a$, of weak interchain bonds add coherently such that the polymer fails by breaking of the covalent bond. The direct analogy between Eqs. (15) and (16) is evident. When the interchain structural order is "nematic" (i.e., without precise phase order), the conditions become $L / a \gg\left(t_{0} / t_{3 \mathrm{D}}\right)^{2}$ and $L / a \gg$ $\left(E_{0} / E_{3 \mathrm{D}}\right)^{2}$, respectively (Kivelson and Heeger, 1987). Thus the achievement of high-performance materials with nematic order requires even greater longer-range intrachain coherence.

In fact, for conjugated polymers, $E_{0}$ results from a combination of $\sigma$ and $\pi$ bonds (the latter being equal to $\left.t_{0}\right)$ and $E_{3 \mathrm{D}}$ is dominated by the interchain transfer integral, $t_{3 \mathrm{D}}$. Thus the inequalities imply that, quite generally, the conductivity and the mechanical properties will improve in a correlated manner as the degree of chain alignment is increased. This prediction is in excellent agreement with data obtained from studies of the poly(3-alkylthiophenes), the poly(phenylene vinylenes), poly(thienylene vinylene), and polyacetylene (Heeger and Smith, 1991).

Assuming that the linear correlation persists to even higher draw ratios, the extrapolated modulus of $300 \mathrm{GPa}$ for polyacetylene would imply that the electrical conductivity of perfectly oriented polyacetylene would be approximately $2 \times 10^{5} \mathrm{~S} / \mathrm{cm}$ (Tokito et al., 1991). However, this extrapolated value might still be limited by structural defects (e.g., $s p^{3}$ defects, etc.) rather than by intrinsic phonon scattering.

A theoretical estimate of the intrinsic conductivity, limited by phonon scattering, yields the following expression (Kivelson and Heeger, 1987)

$$
\sigma_{\|}=\left(e^{2} / 2 h a\right) n a^{3}\left(2 \pi M \omega_{0} t_{0}^{2} / \alpha^{2} h\right) \exp \left[h v_{\mathrm{ph}} / k_{B} T\right],
$$

where $\sigma_{\|}$is the conductivity parallel to the chain, $h$ is Planck's constant, $\alpha$ is the strength of the electronphonon interaction, $M$ is the mass of the repeat unit of length $a$, and $t_{0}$ is the transfer integral $(2-3 \mathrm{eV})$. Using parameters obtained for experiment, Eq. (17) predicts $\sigma_{\|}=2 \times 10^{6} \mathrm{~S} / \mathrm{cm}$ at room temperature (Kivelson and Heeger, 1987), more than an order of magnitude greater than that achieved to date. The exponential dependence upon $T$ arises from the fact that backscattering must involve a high-energy phonon with wave number near the zone boundary. The high conductivity values and the exponential increase in conductivity on lowering the temperature predicted by Eq. (17) indicate that the achievement of metallic polymers in which the macromolecules are chain extended and chain aligned is a major opportunity.

\section{The possibility of superconductivity in metallic polymers}

Superconductivity has not yet been observed in doped conjugated polymers. Might one expect superconductivity? If so, what are the materials requirements?

There is every reason to be optimistic that superconductivity will be discovered in doped conjugated polymers:

(i) they are metals;

(ii) the coupling of the electronic structure to the molecular structure is well known. Upon doping, the bond lengths change such that charge is stored in solitons, polarons, and bipolarons. Thus the electron-phonon interaction that is responsible for superconductivity in conventional metals leads to important effects in metallic polymers. In this context, doubly charged bipolarons can be thought of as analogous to real-space Cooper pairs.

As shown above, however, currently available metallic polymers are barely metallic; their electronic properties are dominated by disorder with mean free paths close to the Ioffe-Regel criterion for disorder-induced localization.

It is interesting to compare the transport properties of available metallic polymers with those of the underdoped high- $T_{c}$ superconductors at doping levels where $k_{F} l \sim 1$. For example, when $\mathrm{YBa}_{2} \mathrm{Cu}_{3} \mathrm{O}_{7-\delta}$ is underdoped to values of $\delta$ such that the resistivity increases as the temperature is lowered with $\rho_{r} \equiv \rho(1.4 \mathrm{~K}) / \rho(300 \mathrm{~K}) \approx 2$ (i.e., with temperature dependence similar to that found in the best metallic polymers), the disorder associated with the random occupation of the oxygen sites quenches the superconductivity.

The phase diagrams of $\mathrm{MBa}_{2} \mathrm{Cu}_{3} \mathrm{O}_{7-\delta}$ (where $M=\mathrm{Y}$, Dy, etc.) have been thoroughly studied. For large $\delta$, $\mathrm{MBa}_{2} \mathrm{Cu}_{3} \mathrm{O}_{7-\delta}$ are antiferromagnetic insulators; increasing the oxygen concentration introduces carriers into the $\mathrm{CuO}_{2}$ planes and results in a transition from the antiferromagnetic insulating phase to the metallic and superconducting phases. The changes in resistivity which occur during this evolution from insulator to metal (and superconductor) have been followed in detail in a continuous set of resistivity measurements carried out on ultrathin films (Wang et al., 1991; Yu et al., 1992). Thus the prototypical high- $T_{c}$ "superconductors" show behavior characteristic of the disorder-induced metal-insulator transition. Near the metal-insulator transition, the $\rho$ vs $T$ curves look remarkably similar to those obtained from "metallic" polyaniline, polypyrrole, and PPV. At doping levels near the metal-insulator transition there is no sign whatever of the fact that these copper oxide systems exhibit superconductivity with the highest known transition temperatures.

The point is quite clear. One cannot expect to make any progress toward superconductivity in metallic polymers until there is a major improvement in materials quality to the point where the mean free path is much 
longer than the characteristic monomer repeat units. When this has been achieved, $k_{F} l \gg 1$ and the resistivity will be truly metal-like, decreasing as the temperature decreases. The unresolved question is how to realize the required improvements in structural order. This remains as a major challenge to the field.

Although the required structural order is lacking in materials that are currently available, there is evidence that in metallic polyaniline, the electronic states near the Fermi energy interact strongly and selectively with a specific optical phonon mode (Sariciftci et al., 1994). The $1598-\mathrm{cm}^{-1}$ Raman-active vibrational mode $\left(A_{g}\right.$ symmetry) exhibits a distinct resonance enhancement associated with the mid-IR absorption in metallic polyaniline. Since the mid-IR oscillator strength results from the intraband free-carrier Drude absorption, the resonant enhancement suggests that the symmetry of the electronic wave functions near $E_{F}$ matches the vibrational pattern of the $1598-\mathrm{cm}^{-1}$ normal mode. Quantum chemical calculations of the electronic wave functions and analysis of the normal modes verified that this is in fact true (Sariciftci et al., 1994). Since the coupling of the electronic states near $E_{F}$ to lattice vibrations is known to lead to superconductivity in metals, the demonstration of resonant coupling to a specific vibrational mode provides some optimism; at least this is the kind of feature that one would like to see.

The story of superconductivity in metallic polymers has not yet begun. If and when superconductivity is discovered in this class of materials, that discovery will create a new research opportunity that will be both exciting and potentially important.

\section{APPLICATIONS}

Solid-state photonic devices are a class of devices in which the quantum of light, the photon, plays a role. They function by utilizing the electro-optical and/or optoelectronic effects in solid-state materials. Because the interband optical transition (absorption and/or emission) is involved in photonic phenomena and because photon energies from near infrared (IR) to near ultraviolet (UV) are of interest, the relevant materials are semiconductors with band gaps in the range from 1 to 3 $\mathrm{eV}$. Typical inorganic semiconductors used for photonic devices are Si, Ge, GaAs, GaP, GaN, and SiC. Photonic devices are often classified into three categories: light sources (light-emitting diodes, diode lasers, etc.), photodetectors (photoconductors, photodiodes, etc.) and energy conversion devices (photovoltaic cells). All three are important. Because photonic devices are utilized in a wide range of applications, they continue to provide a focus for research laboratories all over the world.

Most of the photonic phenomena known in conventional inorganic semiconductors have been observed in these semiconducting polymers (Yu and Heeger, 1996). The dream of using such materials in high-performance "plastic" photonic devices is rapidly becoming reality: High-performance photonic devices fabricated from conjugated polymers have been demonstrated, including light-emitting diodes, light-emitting electrochemical cells, photovoltaic cells, photodetectors, and optocouplers; i.e., all the categories which characterize the field of photonic devices. These polymer-based devices have reached performance levels comparable to or even better than their inorganic counterparts.

This Nobel Lecture is, perhaps, not the proper place to discuss in detail the many current and anticipated applications of semiconducting and metallic polymers; there is simply neither time in the presentation nor space in the written document to do justice to the subject. Suffice it to say here that I am convinced that we are on the verge of a revolution in "plastic electronics." I refer those interested to recent reviews that focus on these important developments (McGehee and Heeger, 2000; McGhee et al., 1999).

The advances in printing technology, for example, high-resolution ink-jet printing, to fabricate semiconductor devices by processing the conducting polymer from solution has proven to be an enabling step in the development of plastic electronic devices such as diodes, photodiodes, LED's, LEC's, optocouplers, and thin-film transistors (Yu and Heeger, 1996).

\section{CONCLUDING COMMENTS}

I am greatly honored to receive the Nobel Prize in Chemistry for "the discovery and development of conducting polymers." The science of semiconducting and metallic polymers is inherently interdisciplinary; it falls at the intersection of chemistry and physics. In creating and expanding this "fourth generation" of polymers, we attempted to understand nature with sufficient depth that we could achieve materials with novel and unique properties, properties that are not otherwise available. This was (and is) an elegant and somewhat dangerous exercise; elegant because it required the synthesis of knowledge from the two disciplines, and dangerous because one is always pushing beyond the knowledge and experience of his background. I started out as a physicist; however, I am what I have become. I have evolved, with the help of many colleagues in the international scientific community, into an interdisciplinary scientist. That my work and that of my colleagues Alan MacDiarmid and Hideki Shirakawa has had sufficient impact on chemistry to be recognized by the Nobel Prize gives $\mathrm{me}$, therefore, particular satisfaction.

\section{REFERENCES}

Abrahams, E., P. W. Anderson, D. C. Licciardello, and T. V. Ramakrishnan, 1979, Phys. Rev. Lett. 42, 695.

Akagi, K., M. Suezaki, H. Shirakawa, H. Kyotani, M. Shimamura, and Y. Tanabe, 1989, Synth. Met. 28, D1.

Allemand, P. M., G. Srdanov, A. Koch, and F. Wudl, 1991, J. Am. Chem. Soc. 113, 1050.

Anderson, P. W., 1958, Phys. Rev. 109, 1492.

Anderson, P. W., 1970, Comments Solid State Phys. 2, 193.

Brazovskii, S. A., and N. Kirova, 1981, JETP Lett. 33, 4. 
Brazovskii, S., N. Kirova, and A. R. Bishop, 1998, Opt. Mater. 9, 465 .

Burroughes, J. H., D. D. C. Bradley, A. R. Brown, R. N. Marks, R. H. Friend, P. L. Burns, and A. B. Holmes, 1990, Nature (London) 347, 539.

Burroughes, J. H., C. A. Jones, and R. H. Friend, 1988, Nature (London) 335, 137.

Campbell, I. H., T. W. Hagler, D. L. Smith, and J. P. Ferraris, 1996, Phys. Rev. Lett. 76, 1900.

Cao, Y., P. Smith, and A. J. Heeger, 1991, Polymer 32, 1210.

Cao, Y., P. Smith, and A. J. Heeger, 1992, Synth. Met. 48, 91.

Cao, Y., P. Smith, and A. J. Heeger, U.S. patent 5,232,631.

Cao, Y., G. M. Treacy, P. Smith, and A. J. Heeger, 1992, Appl. Phys. Lett. 60, 2711.

Cao, Y., G. Yu, C. Zhang, R. Menon, and A. J. Heeger, 1997, Synth. Met. 87, 171.

Castner, T. G., 1991, in Hopping Transport in Solids, edited by M. Pollak and B. I. Shlovskii (Elsevier Science, Amsterdam), p. 1.

Chiang, C. K., C. R. Fincher, Jr., Y. W. Park, A. J. Heeger, H. Shirakawa, and E. J. Louis, 1977, Phys. Rev. Lett. 39, 1098.

Chiang, C. K., S. C. Gau, C. R. Fincher, Jr., Y. W. Park, and A. G. MacDiarmid, 1978, Appl. Phys. Lett. 33, 18.

Chiang L. Y., A. F. Garito, and D. J. Sandman, eds., 1992, in Electrical, Optical, and Magnetic Properties of Organic Solid State Materials, Mater. Res. Soc. Symp. Proc. No. 247 (Materials Research Society, Pittsburgh).

Cornil, J., D. A. do Santos, X. Crispin, R. Silbey, and J. L. Bredas, 1998, J. Am. Chem. Soc. 120, 1289.

Drury, C. J., C. M. J. Mutsaers, C. M. Hart, M. Matters, and D. M. de Leeuw, 1998, Appl. Phys. Lett. 73, 108.

Fesser, K., A. R. Bishop, and D. Campbell, 1983, Phys. Rev. B 27, 4804.

Fincher, C. R., C. E. Chen, A. J. Heeger, A. G. MacDiarmid, and J. B. Hastings, 1982, Phys. Rev. Lett. 48, 100.

Friedrich, J., and K. Werner, U.S. patent 5,300,575.

Gao, J., A. J. Heeger, J. Y. Lee, and C. Y. Kim, 1996, Synth. Met. 82, 221.

Garnier, F., R. Hajlaoui, A. Yasser, and P. Srivastava, 1994, Science 265, 1684.

Gustafsson, G., Y. Cao, G. M. Treacy, F. Klavetter, N. Colaneri, and A. J. Heeger, 1992, Nature (London) 357, 477.

Hagler, T. W., and A. J. Heeger, 1992, Chem. Phys. Lett. 189, 333.

Hagler, T. W., K. Pakbaz, K. Voss, and A. J. Heeger, 1991, Phys. Rev. B 44, 8652.

Halls, J. J. M., C. M. Walsh, N. C. Greenham, E. A. Marseglia, R. H. Friend, S. C. Moratti, and A. B. Holmes, 1995, Nature (London) 376, 498.

Heeger, A. J., 1989, Faraday Discuss. Chem. Soc. 88, 1.

Heeger, A. J., S. Kivelson, J. R. Schrieffer, and W. P. Su, 1988, Rev. Mod. Phys. 60, 781.

Heeger, A. J., and P. Smith, 1991, in Conjugated Polymers, edited by J. L. Bredas and R. Silbey (Kluwer Academic, Dordrecht), p. 141.

Heywang, G., and F. Jonas, 1992, Adv. Mater. 4, 116.

Hide, F., M. Diaz-Garcia, B. Schwartz, M. Andersson, Q. Pei, and A. J. Heeger, 1996, Science 73, 1833.

Ioffe, A. F., and A. R. Regel, 1960, Prog. Semicond. 4, 237. Jenekhe, S. A., and J. A. Osaheni, 1994, Science 265, 765.

Kirova, N., S. Brazovskii, and A. R. Bishop, 1999, Synth. Met. 100, 29.
Kittel, C., 1986, Introduction to Solid State Physics (Wiley, New York).

Kivelson, S., and A. J. Heeger, 1987, Synth. Met. 22, 371.

Kobayashi, M., N. Colaneri, M. Boysel, F. Wudl, and A. J. Heeger, 1985, J. Chem. Phys. 83, 5717.

Kraabel, B. D., J. C. Hummelen, D. Vacar, D. Moses, N. S. Sariciftci, and A. J. Heeger, 1996, J. Chem. Phys. 104, 4267.

Kraabel, B., D. McBranch, N. S. Sariciftci, D. Moses, and A. J. Heeger, 1994, Mol. Cryst. Liq. Cryst. 256, 733.

Lanzani, G., C. Zenz, G. Cerullo, W. Graupner, G. Leising, U. Scherf, and S. De Silvestri, 2000, Synth. Met. 111-112, 493.

Larkin, A. I., and D. E. Khmelnitskii, 1982, Sov. Phys. JETP 56, 647.

Lee, C. H., G. Yu, D. Moses, K. Pakbaz, C. Zhang, N. S. Sariciftci, A. J. Heeger, and F. Wudl, 1993, Phys. Rev. B 48, 15425.

Lee, K., A. J. Heeger, and Y. Cao, 1993, Phys. Rev. B 48, 14884.

Lee, K., A. J. Heeger, and Y. Cao, 1995, Synth. Met. 69, 261.

Lee, K., R. Jansson, N. S. Sariciftci, and A. J. Heeger, 1994, Phys. Rev. B 49, 5781.

Lee, K., R. Menon, C. O. Yoon, and A. J. Heeger, 1995, Phys. Rev. B 52, 4779.

Lee, K., E. K. Miller, A. N. Aleshin, R. Menon, A. J. Heeger, J. H. Kim, C. O. Yoon, and H. Lee, 1998, Adv. Mater. 10, 456. Lee, P. A., and T. V. Ramakrishnan, 1985, Rev. Mod. Phys. 57, 287.

MacDiarmid, A. G., and A. J. Epstein, 1990, in Conjugated Polymeric Materials: Opportunities in Electronics, Optical Electronics and Molecular Electronics, edited by J. L. Bredasand and R. R. Chance (Kluwer Academic, Dordrecht), p. 53.

Maniloff, E. S., D. Vacar, D. W. McBranch, H. Wang, B. R. Mattes, J. Gao, and A. J. Heeger, 1997, Opt. Commun. 141, 243.

McGehee, M. D., and A. J. Heeger, 2000, Adv. Mater. 22, 1655. McGehee, M. D., E. K. Miller, D. Moses, and A. J. Heeger, 1999, in Advances in Synthetic Metals: Twenty Years of Progress in Science and Technology, edited by P. Bernier, S. Lefrant, and G. Bidan (Elsevier Science, Lausanne), p. 98.

McMillan, W. L., 1981, Phys. Rev. B 24, 2739.

Menon, R., C. O. Yoon, D. Moses, and A. J. Heeger, 1998, in Handbook of Conducting Polymers, 2nd ed., edited by T. A. Skotheim, R. L. Elsenbaumer, and J. R. Reynolds (Marcel Dekker, New York), p. 85.

Miller, E. K., C. Y. Yang, and A. J. Heeger, 2000, Phys. Rev. B 62, 6889 .

Miller, E. K., D. Yoshida, C. Y. Yang, and A. J. Heeger, 1999, Phys. Rev. B 59, 4661.

Morita, S., A. A. Zakhidov, and Y. Yoshino, 1992, Solid State Commun. 82, 249.

Mott, N. F., 1990, Metal-Insulator Transition (Taylor \& Francis, London).

Mott, N. F., and E. A. Davis, 1979, Electronic Processes in Noncrystalline Materials (Oxford University, Oxford).

Mott, N. F., and M. Kaveh, 1985, Adv. Phys. 34, 329.

Nigrey, P. J., A. G. MacDiarmid, and A. J. Heeger, 1979, Chem. Commun. 96, 594.

Parker, I. D., 1994, J. Appl. Phys. 75, 1656.

Pei, Q., G. Yu, C. Zhang, Y. Yang, and A. J. Heeger, 1995, Science 269, 1086.

Peierls, R. E., 1955, Quantum Theory of Solids (Clarendon, Oxford). 
Pope, M., and C. E. Swenberg, 1982, Electronic Processes in Organic Crystals (Clarendon, Oxford).

Rånby, B., 1993, in Conjugated Polymers and Related Materials: The Interconnection of Chemical and Electronic Structures, edited by W. R. Salaneck, I. Lundström, and B. Rånby (Oxford University, Oxford), Chap. 3.

Reghu, M., Y. Cao, D. Moses, and A. J. Heeger, 1993, Phys. Rev. B 497, 1758.

Rothberg, L. J., M. Yan, F. Papadimitrakopoulos, M. E. Galvin, E. W. Kwock, and T. M. Miller, 1996, Synth. Met. 80, 41.

Salaneck, W. R., I. Lundstrom, W. S. Haung, and A. G. MacDiarmid, 1986, Synth. Met. 13, 291.

Sariciftci, N. S., 1998, Primary Photoexcitations in Conjugated Polymers (World Scientific, Singapore).

Sariciftci, N. S., and A. J. Heeger, 1994, Int. J. Mod. Phys. B 8, 237.

Sariciftci, N. S., A. J. Heeger, V. Krasevec, P. Venturini, D. Mihailovic, Y. Cao, J. Libert, and J. L. Bredas, 1994, Synth. Met. 62, 107.

Sariciftci, N. S., L. Smilowitz, A. J. Heeger, and F. Wudl, 1992, Science 258, 1474.

Schimmel, T., W. Reiss, J. Gmeiner, M. Schworer, H. Naarmann, and N. Theophilou, 1998, Solid State Commun. 65, 1311.

Shirakawa, H., E. J. Louis, A. G. MacDiarmid, C. K. Chiang, and A. J. Heeger, 1977, Chem. Commun. 578.

Smilowitz, L., N. S. Sariciftci, R. Wu, C. Gettinger, A. J. Heeger, and F. Wudl, 1993, Phys. Rev. B 47, 13835.

Smith, P., P. J. Lemstra, J. P. L. Pijpers, and A. M. Kile, 1981, Colloid Polym. Sci. 259, 1070.

Streitweiser, A., 1961, Molecular Orbital Theory for Organic Chemists (Wiley, New York).

Su, W. P., J. R. Schrieffer, and A. J. Heeger, 1979, Phys. Rev. Lett. 42, 1698.
Su, W. P., J. R. Schrieffer, and A. J. Heeger, 1980, Phys. Rev. B 22, 2099.

Sun, L., and S. C. Yang, 1992, Polym. Prepr. (Am. Chem. Soc. Div. Polym. Chem.) 33, 379.

Tokito, S., P. Smith, and A. J. Heeger, 1991, Polymer 32, 464. Tomozawa, H., D. Braun, S. Philips, A. J. Heeger, and H. Kroemer, 1987, Synth. Met. 22, 63.

Tomozawa, H., D. Braun, S. D. Philips, R. Worland, and A. J. Heeger, 1989, Synth. Met. 28, C687.

Tranquada, J. M., 1990, in Earlier and Recent Aspects of Superconductivity, edited by J. G. Bednorz and K. A. Muller (Springer, Berlin), p. 422.

Tsukomoto, J., 1990, Jpn. J. Appl. Phys., Part 1 29, 1.

Wang, T., K. M. Beauchamp, D. D. Berkley, B. R. Johnson, J. X. Liu, J. Zhang, and A. M. Goldman, 1991, Phys. Rev. B 43, 8623.

Wudl, F., R. O. Angus, F. L. Lu, P. M. Allemand, D. J. Vachon, M. Nowak, Z. X. Liu, and A. J. Heeger, 1987, J. Am. Chem. Soc. 109, 3677.

Yang, C. Y., Y. Cao, P. Smith, and A. J. Heeger, 1992, Synth. Met. 53, 293.

Yang, C. Y., and A. J. Heeger, 1996, Synth. Met. 83, 85. Yannoni, C. S., and T. C. Clarke, 1983, Phys. Rev. Lett. 52, 1191.

Yu, G., J. Gao, J. C. Hummelen, F. Wudl, and A. J. Heeger, 1995, Science 270, 1789.

Yu, G., and A. J. Heeger, 1995, J. Appl. Phys. 78, 4510.

Yu, G., and A. J. Heeger, 1996, in The Physics of Semiconductors, edited by M. Schleffer and R. Zimmerman (World Scientific, Singapore), Vol. 1, p. 35.

Yu, G., C. H. Lee, A. J. Heeger, N. Herron, E. M. McCarron, L. Cong, G. C. Spalding, C. A. Nordman, and A. M. Goldman, 1992, Phys. Rev. B 45, 4964. 